\title{
Closed Form Pricing Formulas for Discretely Sampled Generalized Variance Swaps
}

\author{
Wendong Zheng and Yue Kuen Kwok* \\ Department of Mathematics \\ Hong Kong University of Science and Technology \\ Clear Water Bay, Hong Kong
}

\begin{abstract}
Most of the existing pricing models of variance derivative products assume continuous sampling of the realized variance processes, though actual contractual specifications compute the realized variance based on sampling at discrete times. We present a general analytic approach for pricing discretely sampled generalized variance swaps under the stochastic volatility models with simultaneous jumps in the asset price and variance processes. The resulting pricing formula of the gamma swap is in closed form while those of the corridor variance swaps and conditional variance swaps take the form of onedimensional Fourier integrals. We also verify through analytic calculations the convergence of the asymptotic limit of the pricing formulas of the discretely sampled generalized variance swaps under vanishing sampling interval to the analytic pricing formulas of the continuously sampled counterparts. The proposed methodology can be applied to any affine model and other higher moments swaps as well. We examine the exposure to convexity (volatility of variance) and skew (correlation between the equity returns and variance process) of these discretely sampled generalized variance swaps. We explore the impact on the fair strike prices of these exotic variance swaps with respect to different sets of parameter values, like varying sampling frequencies, jump intensity, and width of the monitoring corridor.
\end{abstract}

Key words: generalized variance swaps, stochastic volatility models, Fourier transform, discrete sampling

${ }^{*}$ Correspondence author; e-mail: maykwok@ust.hk 


\section{Introduction}

Volatility measures the standard deviation of the logarithm of returns of an underlying asset, thus it gives a measure of the risk of holding that asset. Volatility risk has drawn a wider attention in the financial markets in recent years, especially after the global financial crisis. Volatility trading becomes an important topic of risk management. In a bearish market environment, volatility typically stays at a high level, so holding a long position of volatility may be useful in hedging an equity portfolio. Indeed, volatility can be viewed as an asset class in its own right. Investors can use volatility derivatives to perform directional trading of volatility levels, say, trading the spread between the realized and implied volatility levels, or hedging an implicit volatility exposure. These volatility derivatives are investment tools for investors with specific views on the future market volatility or with particular risk exposures by allowing them to deal with these views or risks without taking a direct position in the underlying asset and/or delta-hedging their position (Brockhaus and Long, 2000).

Volatility products can be generally classified into two types. The historical-variance-based volatility derivatives include products whose payoff depends on the realized variance of the underlying asset. Another class of volatility products are the implied-volatility-based products, like the VIX futures traded in the Chicago Board Options Exchange (CBOE). The VIX stands for the CBOE Volatility Index, and it measures the 30-day expected future volatility of the S\&P 500 index (Carr and Wu, 2006). In recent years, the third generation of volatility products, which are known as the generalized variance swaps, including the corridor variance swaps, conditional variance swaps and gamma swaps, have gained wider popularity as volatility trading instruments. These exotic variance swaps can offer investors a more finely tuned volatility exposure than the traditional variance swaps [see the review articles by Carr and Lewis (2004), Bouzoubaa and Osseiran (2010) and Lee (2010)]. Their product specifications and potential uses in hedging or betting the various forms of volatility exposures will be presented in later sections. One of the objectives of this paper is to present a systematic and efficient analytic approach for pricing these discretely sampled generalized variance swaps under the stochastic volatility models with simultaneous jumps in the asset price and variance processes.

Assuming that the stock prices evolve without jumps, Neuberger (1994) shows how a continuously sampled variance swap can be theoretically equivalent to a dynamically adjusted constant dollar exposure to the stock, in combination with a static long position in a portfolio of options and a forward contract that replicate the payoff of a log contract. Carr and Madan (1998) propose various methods of trading the realized volatility, like taking a static position in options, delta-hedging an option position, etc. They demonstrate that the delta-hedged option approach exhibits a large amount of path dependency in the underlying in the final profit/loss. On the other hand, volatility derivatives are shown to provide the pure exposure to realized

market volatility without an inherent price path dependency. Demeterfi et al. (1999) provide 
a nice review on the pricing behavior and theory of both variance and volatility swaps.

As a common approximation assumption in the pricing models of volatility derivatives in the literature, the discretely sampled realized variance in the actual contractual specification is approximated by a continuously sampled variance (as quantified by the quadratic variation of the log asset price process). For some volatility products, it occurs that the assumption of continuous sampling falls short of providing pricing results with sufficient accuracy when the actual discrete sampling becomes less frequent. Since it is not so straightforward to estimate the approximation errors in a unified framework, practitioners trading on contracts that are based on the realized variance with a low sampling frequency cannot properly assess the pricing errors caused by the continuous sampling assumption. A review on the replication errors for the discretely monitored variance swaps can be found in Carr and Lee (2009). Keller-Ressel and Muhle-Karbe (2010) discuss the rate of convergence of the approximation of the realized variance via the notion of quadratic variation and examine the errors of the approximation in pricing short-dated options with non-linear payoffs.

There have been numerous papers that consider pricing variance product contracts on the discretely sampled realized variance. Little and Pant (2001) develop a finite difference approach for the valuation of the discretely sampled variance swaps in an extended Black-Scholes framework with a local volatility function. They adopt an effective numerical technique to capture the jumps in the realized variance across the sampling dates. Windcliff et al. (2006) improve on the pricing algorithm for the discretely sampled volatility derivatives by allowing jumps in the asset price process. Using the Monte Carlo simulation method, Broadie and Jain (2008) investigate the effect of discrete sampling and asset price jumps on the fair strike prices of variance and volatility swaps under various stochastic volatility models. Carr and Lee (2009) consider the replication of discretely sampled variance products (including exotic path dependent payoff structures) using options, futures, and bonds with the same sampling frequency of the variance products. Itkin and Carr (2010) use a forward characteristic function approach to price discretely monitored variance and volatility swaps under various Lévy models with stochastic time change. Crosby and Davis (2011) consider the pricing of generalized variance swaps, such as self-quantoed variance swaps, gamma swaps, skewness swaps and proportional variance swaps under the time-changed Lévy processes. They show that the prices of discretely monitored variance swaps and their generalizations all converge to the prices of continuously monitored counterparts as $O(1 / N)$, where $N$ is the number of monitoring instants. Sepp (2011) analyzes the impact of discrete sampling on the pricing of options on the realized variance under Heston's stochastic volatility model. He proposes a method of mixing the discrete variance in a log-normal model and the quadratic variance in a stochastic volatility model that approximates well the distribution of the discrete variance. Drimus and Farkas (2010) show that conditioning on the realization of the instantaneous variance process, the residual randomness arising from discrete sampling follows a normal distribution. They also 
provide a practical analysis of the greeks sensitivity of options on discretely sampled variance. Following the Little-Pant pricing formulation, Zhu and Lian (2011) manage to derive closed form pricing formulas for the vanilla variance swaps under Heston's stochastic volatility model for the underlying asset price process by solving a coupled system of partial differential equations. Lian (2010) extends the above analytic pricing approach to the underlying asset price process that allows stochastic volatility with simultaneous jumps in both the asset price and variance process (SVSJ model). The success of analytic tractability in the Zhu-Lian approach lies on the exponential affine structure of the SVSJ model, where the corresponding analytic formulas of the marginal characteristic functions can be derived. When the payoff structures of the variance swap contracts become more exotic, like those of the corridor variance swaps, conditional variance swaps and gamma swaps, the knowledge of the marginal characteristic functions alone may not be enough in the derivation of the corresponding closed form pricing formulas. Instead, the joint moment generating function plays a vital role in deriving the pricing formulas for exotic variance swaps.

In this paper, we propose a general analytic approach for pricing various types of discretely sampled generalized variance swaps, thanks to the availability of the analytical expression of the joint moment generating function of the underlying processes. The analytic derivation of the associated moment generating function under the SVSJ model can be accomplished via the solution to a Riccati system of ordinary differential equations. Provided that the payoff function of a generalized variance swap can be transformed into an exponential function of the state variables, closed form or semi-analytic (in terms of one-dimensional Fourier integrals) pricing formula of the derivative product can be derived. Duffie et al. (2000) and Chacko and Das (2002) demonstrate the versatility of this analytic approach in pricing various types of fixed income derivatives. These papers explore various invariant properties of the solutions to the Riccati systems and manage to express the pricing formulas in terms of these solutions. Sepp (2007) applies similar techniques to price continuously-sampled variance derivatives and conditional variance swaps via the derivation of the analytic representation of the Green function associated with the governing partial integro-differential equation under the SVSJ model.

This paper is organized as follows. In the next section, we present the formulation of the SVSJ model with a discussion on various possible extensions of the underlying joint dynamics of the asset returns and its variance. Thanks to the exponential affine structures of the SVSJ model, we manage to obtain an analytic representation of the corresponding joint moment generating function by solving a Riccati system of ordinary differential equations. In Section 3 , we present the product specification and potential uses of various generalized variance swaps. We then show how to derive the closed form pricing formula of each of these discretely sampled generalized variance swaps under the SVSJ model. The continuously sampled gamma swap is known to provide a constant share gamma exposure. We illustrate how this gamma exposure 
property is modified under discrete sampling. The pricing of the conditional variance swap requires the computation of the expected occupation time of the asset price within a specified corridor. For the formulas of the fair strikes of the discretely monitored variance swaps and gamma swaps, we take the asymptotic limit by letting the sampling interval approach zero and illustrate that one can recover the same set of formulas of their continuously sampled counterparts (Sepp, 2008). Also, we manage to obtain the pricing formulas of corridor and conditional variance swaps under continuous sampling. In Section 4, we report the numerical tests that examine the convergence of the fair strike prices with increasing sampling frequencies to the fair strike price of the continuously sampled counterparts. Our numerical tests show that an almost linear rate of convergence with respect to the sampling interval of the fair strike price under discrete sampling to that under continuously sampling is revealed for variance swaps. However, such convergence behavior may not always be observed for other exotic variance swaps. We also examine the exposure to convexity (volatility of variance), skew (correlation between equity returns and variance process) and jump intensities of these exotic swap products under the more realistic framework of discrete sampling of the realized variance. The fair strike prices in these generalized variance swaps are shown to be dependent on their contractual specifications. Summary and conclusive remarks are presented in the last section.

\section{Stochastic volatility models with simultaneous jumps and joint moment generating function}

There have been numerous empirical studies on the dynamics of asset returns that illustrate evidence for both jumps in the price level and its volatility. A prominent continuous time model that has been widely adopted is the affine simultaneous jump model (Duffie et al., 2000) where the asset return and its variance follow the jump-diffusion process for which the drift, covariance and jump intensities are assumed to have an affine dependence on the state vector. The analytic pricing under the affine simultaneous jump model can be performed by solving the corresponding Riccati system of ordinary differential equations (Chacko and Das, 2002).

In this paper, we adopt the following stochastic volatility model with simultaneous jumps (SVSJ) to describe the joint dynamics of the stock price $S_{t}$ and its instantaneous variance $V_{t}$. Under the risk neutral pricing measure $Q$, the joint dynamics of $S_{t}$ and $V_{t}$ assumes the form

$$
\left\{\begin{aligned}
\frac{\mathrm{d} S_{t}}{S_{t}} & =(r-d-\lambda m) \mathrm{d} t+\sqrt{V_{t}} \mathrm{~d} W_{t}^{S}+\left(e^{J^{S}}-1\right) \mathrm{d} N_{t} \\
\mathrm{~d} V_{t} & =\kappa\left(\theta-V_{t}\right) \mathrm{d} t+\varepsilon \sqrt{V_{t}} \mathrm{~d} W_{t}^{V}+J^{V} \mathrm{~d} N_{t}
\end{aligned}\right.
$$

where $W_{t}^{S}$ and $W_{t}^{V}$ are a pair of correlated standard Brownian motions with $\mathrm{d} W_{t}^{S} \mathrm{~d} W_{t}^{V}=\rho \mathrm{d} t$, and $N_{t}$ is a Poisson process with constant intensity $\lambda$ that is independent of the two Brownian 
motions. Here, $\rho$ is the constant correlation coefficient. We let $J^{S}$ and $J^{V}$ denote the random jump size of the log price and variance, respectively, and these random jump sizes are assumed to be independent of $W_{t}^{S}, W_{t}^{V}$ and $N_{t}$. Also, we let $r$ and $d$ denote the riskless interest rate and the constant dividend yield, respectively, and $m=E_{t}^{Q}\left[e^{J^{S}}-1\right]$. Throughout the paper, all the expectation calculations $E_{t}^{Q}[\cdot]$ are performed under the risk neutral pricing measure $Q$ and conditional on filtration $\mathcal{F}_{t}$ at the current time $t$. In the sequel, we suppress the superscript and subscript in the expectation operator for notational convenience.

It is well known that jumps in the stock price provide a more realistic description of the short term behavior of the stock price dynamics while jumps in the variance give the more accurate modeling of the volatility skew. Various empirical studies reveal that jumps in the price level and variance in general occur together, and they are strongly interdependent and have opposite sign. One may argue that the above SVSJ model with specific affine forms of the parameter functions may be somewhat restrictive. Some recent non-parametric studies of the high frequency movements in stock market volatility reveal that volatility may follow quite different forms of jump behavior (Todorov and Tauchen, 2010). Various extended versions of the stochastic volatility models have also been proposed. For example, Kangro et al. (2004) propose to generalize the intensity of the Poisson process to be a non-reverting stochastic process. Carr and $\mathrm{Wu}$ (2007) assume stochastic hazard rate for the Poisson process, where the stochastic hazard rate parameter is assumed to be the sum of the instantaneous variance and a latent risk factor that follows a diffusion process with mean reversion drift rate. Cont and Kokholm (2008) model directly the forward variance swap rates for a discrete tenor of maturities, somewhat analogous to the LIBOR market model in interest rates modeling. In some of these extended models, analytic tractability that is similar to the SVSJ model can be maintained though the analytic procedures tend to become more involved. In this paper, we illustrate the set of analytic procedures of deriving the pricing formulas of discretely sampled exotic variance products under the popular SVSJ model and relegate the research on analytic pricing methods under other types of stochastic volatility models to future works.

\section{Joint moment generating function}

For convenience, we let $X_{t}=\ln S_{t}$. The joint moment generating function of the joint process $X_{t}$ and $V_{t}$ is defined to be

$$
E\left[\exp \left(\phi X_{T}+b V_{T}+\gamma\right)\right]
$$

where $\phi, b$ and $\gamma$ are constant parameters. Let $U\left(X_{t}, V_{t}, t\right)$ denote the non-discounted time- $t$ value of a contingent claim with the terminal payoff function: $U\left(X_{T}, V_{T}, T\right)$, where $T$ is the maturity date. By adopting the temporal variable, $\tau=T-t$, it can be deduced from the Feynman-Kac theorem that $U(X, V, \tau)$ is governed by the following partial integro-differential 
equation (PIDE):

$$
\begin{aligned}
\frac{\partial U}{\partial \tau}= & \left(r-d-m \lambda-\frac{V}{2}\right) \frac{\partial U}{\partial X}+\kappa(\theta-V) \frac{\partial U}{\partial V}+\frac{V}{2} \frac{\partial^{2} U}{\partial X^{2}}+\frac{\varepsilon^{2} V}{2} \frac{\partial^{2} U}{\partial V^{2}}+\rho \varepsilon V \frac{\partial^{2} U}{\partial X \partial V} \\
& +\lambda E\left[U\left(X+J^{S}, V+J^{V}, \tau\right)-U(X, V, \tau)\right] .
\end{aligned}
$$

The terminal payoff function of the contingent claim becomes the initial condition of the PIDE. Note that the joint moment generating function (MGF) can be regarded as the time- $t$ forward value of the contingent claim with the terminal payoff: $\exp \left(\phi X_{T}+b V_{T}+\gamma\right)$, so the MGF also satisfies PIDE (2.2).

Using the analytic procedures similar to those in interest rate derivatives pricing under the stochastic volatility with simultaneous jumps model (Chacko and Das, 2002), analytic solution to the joint MGF can be obtained by solving a Riccati system of ordinary differential equations. Due to the exotic payoff structure of the generalized variance swaps, analytic form of the joint MGF is required in deriving analytic pricing formulas for these discretely sampled variance products. As a remark, it suffices to use the marginal MGFs to price discretely sampled vanilla variance swaps (Zhu and Lian, 2011) due to their simpler payoff structure. Once the joint MGF is known, the respective marginal MGF can be obtained easily by setting the irrelevant parameters in the joint MGF to be zero. For example, the marginal MGF with respect to the state variable $V$ can be obtained by setting $\phi=\gamma=0$.

Thanks to the affine structure in the SVSJ model, $U(X, V, \tau)$ admits an analytic solution of the following form (Duffie et al., 2002):

$$
U(X, V, \tau)=\exp (\phi X+B(\Theta ; \tau, \mathbf{q}) V+\Gamma(\Theta ; \tau, \mathbf{q})+\Lambda(\Theta ; \tau, \mathbf{q}))
$$

where the parameter functions $B(\Theta ; \tau, \mathbf{q}), \Gamma(\Theta ; \tau, \mathbf{q})$ and $\Lambda(\Theta ; \tau, \mathbf{q})$ satisfy the following Riccati system of ordinary differential equations:

$$
\left\{\begin{array}{l}
\frac{\partial B}{\partial \tau}=-\frac{1}{2}\left(\phi-\phi^{2}\right)-(\kappa-\rho \varepsilon \phi) B+\frac{\varepsilon^{2}}{2} B^{2} \\
\frac{\partial \Gamma}{\partial \tau}=(r-d) \phi+\kappa \theta B \\
\frac{\partial \Lambda}{\partial \tau}=\lambda\left(E\left[\exp \left(\phi J^{S}+B J^{V}\right)-1\right]-m \phi\right)
\end{array}\right.
$$

with the initial conditions: $B(0)=b, \Gamma(0)=\gamma$ and $\Lambda(0)=0$. Here, $\mathbf{q}=(\phi b \gamma)^{T}$ and we use $\Theta$ to indicate the dependence of these parameter functions on the model parameters in the SVSJ model. One has to specify the distributions for $J^{S}$ and $J^{V}$ in order to obtain a complete solution to these parameter functions. For example, suppose we assume that $J^{V} \sim \exp (1 / \eta)$ (exponential distribution with parameter rate $1 / \eta$ ) and $J^{S}$ follows

$$
J^{S} \mid J^{V} \sim \operatorname{Normal}\left(\nu+\rho_{J} J^{V}, \delta^{2}\right)
$$

that is the Gaussian distribution with mean $\nu+\rho_{J} J^{V}$ and variance $\delta^{2}$, we obtain

$$
m=E\left[e^{J^{S}}-1\right]=\frac{e^{\nu+\delta^{2} / 2}}{1-\eta \rho_{J}}-1,
$$


provided that $\eta \rho_{J}<1$. Under the above assumption on $J^{S}$ and $J^{V}$, the parameter functions can be found to be

$$
\left\{\begin{aligned}
B(\Theta ; \tau, \mathbf{q})= & \frac{b\left(\xi_{-} e^{-\zeta \tau}+\xi_{+}\right)-\left(\phi-\phi^{2}\right)\left(1-e^{-\zeta \tau}\right)}{\left(\xi_{+}+\varepsilon^{2} b\right) e^{-\zeta \tau}+\xi_{-}-\varepsilon^{2} b} \\
\Gamma(\Theta ; \tau, \mathbf{q})= & (r-d) \phi \tau+\gamma-\frac{\kappa \theta}{\varepsilon^{2}}\left[\xi_{+} \tau+2 \ln \frac{\left(\xi_{+}+\varepsilon^{2} b\right) e^{-\zeta \tau}+\xi_{-}-\varepsilon^{2} b}{2 \zeta}\right] \\
\Lambda(\Theta ; \tau, \mathbf{q})= & -\lambda(m \phi+1) \tau+\lambda e^{\phi \nu+\delta^{2} \phi^{2} / 2} \\
& {\left[\frac{k_{2}}{k_{4}} \tau-\frac{1}{\zeta}\left(\frac{k_{1}}{k_{3}}-\frac{k_{2}}{k_{4}}\right) \ln \frac{k_{3} e^{-\zeta \tau}+k_{4}}{k_{3}+k_{4}}\right] }
\end{aligned}\right.
$$

with $\mathbf{q}=(\phi b \gamma)^{T}$ and

$$
\begin{aligned}
\zeta & =\sqrt{(\kappa-\rho \varepsilon \phi)^{2}+\varepsilon^{2}\left(\phi-\phi^{2}\right)}, \\
\xi_{ \pm} & =\zeta \mp(\kappa-\rho \varepsilon \phi), \\
k_{1} & =\xi_{+}+\varepsilon^{2} b, \\
k_{2} & =\xi_{-}-\varepsilon^{2} b, \\
k_{3} & =\left(1-\phi \rho_{J} \eta\right) k_{1}-\eta\left(\phi-\phi^{2}+\xi_{-} b\right), \\
k_{4} & =\left(1-\phi \rho_{J} \eta\right) k_{2}+\eta\left(\phi-\phi^{2}-\xi_{+} b\right) .
\end{aligned}
$$

The derivation of the parameter functions in eq. (2.6) is provided in Appendix A. Once the joint MGF is available, an effective analytic pricing approach can be constructed to derive the pricing formulas of the various types of discretely sampled generalized variance swaps. As an illustration, we demonstrate how the pricing formula of the vanilla variance swap can be readily derived. Let the sampling dates be denoted by $0=t_{0}<t_{1}<\cdots<t_{N}=T$. On the maturity date $T$, the payoff of the vanilla variance swap is defined to be

$$
\frac{A}{N} \sum_{k=1}^{N}\left(\ln \frac{S_{t_{k}}}{S_{t_{k-1}}}\right)^{2}-K
$$

where $K$ is the strike price of the variance swap and $A$ is the annualized factor (say, we take $A=252$ for daily sampling). We write $\Delta t_{k}=t_{k}-t_{k-1}$ and express the time interval $t_{k-1}-t_{0}$ simply as $t_{k-1}$ since $t_{0}$ is taken to be zero. The pricing problem amounts to finding the fair strike price $K$ such that the value of the vanilla variance swap at initiation is zero. The fair strike price $K$ is then given by

$$
K=E\left[\frac{A}{N} \sum_{k=1}^{N}\left(\ln \frac{S_{t_{k}}}{S_{t_{k-1}}}\right)^{2}\right] .
$$

We now show how to evaluate each term in the above summation. Using the known analytic expression of the marginal MGFs, we apply the tower rule in conditional expectation to obtain 
the expectation of a typical term as follows;

$$
\begin{aligned}
E\left[\left(\ln \frac{S_{t_{k}}}{S_{t_{k-1}}}\right)^{2}\right] & =\left.E\left[\frac{\partial^{2}}{\partial \phi^{2}} e^{\phi\left(X_{t_{k}}-X_{t_{k-1}}\right)}\right]\right|_{\phi=0} \\
& =\left.\frac{\partial^{2}}{\partial \phi^{2}} E\left[E\left[e^{\phi X_{t_{k}}} \mid X_{t_{k-1}}, V_{t_{k-1}}\right] e^{-\phi X_{t_{k-1}}}\right]\right|_{\phi=0} \\
& =\left.\frac{\partial^{2}}{\partial \phi^{2}} E\left[e^{B\left(\Theta ; \Delta t_{k}, \mathbf{q}_{1}\right) V_{t_{k-1}}+\Gamma\left(\Theta ; \Delta t_{k}, \mathbf{q}_{1}\right)+\Lambda\left(\Theta ; \Delta t_{k}, \mathbf{q}_{1}\right)}\right]\right|_{\phi=0} \\
& =\left.\frac{\partial^{2}}{\partial \phi^{2}} e^{B\left(\Theta ; t_{k-1}, \mathbf{q}_{2}\right) V_{0}+\Gamma\left(\Theta ; t_{k-1}, \mathbf{q}_{2}\right)+\Lambda\left(\Theta ; t_{k-1}, \mathbf{q}_{2}\right)}\right|_{\phi=0},
\end{aligned}
$$

where $\mathbf{q}_{1}=\left(\begin{array}{lll}\phi & 0 & 0\end{array}\right)^{T}$, and

$$
\mathbf{q}_{2}=\left(\begin{array}{c}
0 \\
B\left(\Theta ; \Delta t_{k}, \mathbf{q}_{1}\right) \\
\Gamma\left(\Theta ; \Delta t_{k}, \mathbf{q}_{1}\right)+\Lambda\left(\Theta ; \Delta t_{k}, \mathbf{q}_{1}\right)
\end{array}\right) .
$$

The fair strike price of the variance swap is then given by

$$
K_{V}(T, N)=\left.\frac{A}{N} \sum_{k=1}^{N} \frac{\partial^{2}}{\partial \phi^{2}} e^{B\left(\Theta ; t_{k-1}, \mathbf{q}_{2}\right) V_{0}+\Gamma\left(\Theta ; t_{k-1}, \mathbf{q}_{2}\right)+\Lambda\left(\Theta ; t_{k-1}, \mathbf{q}_{2}\right)}\right|_{\phi=0} .
$$

Note that the derivation procedure is less involved compared to the pricing approach used by Zhu and Lian (2011).

\section{Asymptotic limit of vanishing sampling interval}

It would be instructive to examine whether we can deduce the formula for the fair strike price of the continuously sampled variance swap by taking the asymptotic limit as $\Delta t \rightarrow 0$, where $\Delta t=\max _{k} \Delta t_{k}$, in formula (2.7). By expanding the parameter functions $B, \Gamma$ and $\Lambda$ in powers of $\Delta t_{k}$ and taking $\Delta t \rightarrow 0$ subsequently, we manage to obtain the following closed form formula for the fair strike of the continuously sampled variance swap

$$
\begin{aligned}
K_{V}(T, \infty)= & \frac{1}{T}\left\{\frac{1-e^{-\kappa T}}{\kappa} V_{0}-\frac{\lambda \eta}{\kappa^{2}}\left(1-e^{-\kappa T}-\kappa T\right)\right. \\
& \left.+\lambda\left[\delta^{2}+\rho_{J}^{2} \eta^{2}+\left(\nu+\rho_{J} \eta\right)^{2}\right] T+\frac{\theta}{\kappa}\left(\kappa T-1+e^{-\kappa T}\right)\right\},
\end{aligned}
$$

where we have used the convention $\frac{A}{N}=\frac{1}{T}$. The above formula is in agreement with a similar pricing formula in Sepp (2008). The proof of formula 2.8 is presented in Appendix B.

In the next section, we illustrate how to generalize the above pricing approach to find the pricing formulas for the various types of generalized variance swaps.

\section{$3 \quad$ Generalized variance swaps}

Vanilla variance swaps are known to be the appropriate instruments to provide investors with pure volatility exposure. In recent years, various types of generalized variance swaps have been 
introduced in the financial markets to enhance volatility trading by providing asymmetric bets or hedges on volatility. Given a tenor structure $\left\{t_{0}, t_{1}, \cdots, t_{N}\right\}$ as before, the generalized realized variance over the period $\left[t_{0}, t_{N}\right]$ is defined to be

$$
\frac{A}{N} \sum_{k=1}^{N} w_{k}\left(\ln \frac{S_{t_{k}}}{S_{t_{k-1}}}\right)^{2},
$$

where $w_{k}$ is some discrete weight process chosen so as to target a specific form of volatility exposure. In this section, analytic pricing of discretely sampled gamma swaps, corridor variance swaps and conditional variance swaps would be considered. For each type of these exotic variance products, we start with the description of their product nature and potential uses in volatility trading and hedging.

\subsection{Gamma swaps}

In a gamma swap, the weight $w_{k}$ is chosen to be $\frac{S_{t_{k}}}{S_{t_{0}}}, k=1,2, \cdots, N$. Accordingly, the terminal payoff of the gamma swap is defined by

$$
\frac{A}{N} \sum_{k=1}^{N} \frac{S_{t_{k}}}{S_{t_{0}}}\left(\ln \frac{S_{t_{k}}}{S_{t_{k-1}}}\right)^{2}-K
$$

The motivation of choosing the weight to be the underlying level is to provide the embedded damping of the large downside variance when the stock price falls close to zero. This feature serves to protect the swap seller from crash risk, thus provides an advantage over the noncapped variance swaps. Another motivation is related to variance dispersion trade, which refers to trading the difference between the realized index volatility and the market-cap weighted sum of the realized volatilities of its constituents. Gamma swaps provides better means to trade dispersion than vanilla variance swaps since the risk associated with changes in weights of the constituent stocks over the life of the variance swap is reduced (Jacquier and Slaoui, 2010).

To find the analytic fair strike price of a discretely sampled gamma swap, we compute the expectation of a typical term in the floating leg of the gamma swap as follows:

$$
\begin{aligned}
E\left[\frac{S_{t_{k}}}{S_{t_{0}}}\left(\ln \frac{S_{t_{k}}}{S_{t_{k-1}}}\right)^{2}\right] & =e^{-X_{0}} E\left[e^{\left.X_{t_{k}}-X_{t_{k-1}}\left(X_{t_{k}}-X_{t_{k-1}}\right)^{2} e^{X_{t_{k-1}}}\right]}\right. \\
& =\left.e^{-X_{0}} E\left[\frac{\partial^{2}}{\partial \phi^{2}} e^{\phi\left(X_{t_{k}}-X_{t_{k-1}}\right)+X_{t_{k-1}}}\right]\right|_{\phi=1} \\
& =\left.e^{-X_{0}} \frac{\partial^{2}}{\partial \phi^{2}} E\left[E\left[e^{\phi X_{t_{k}}} \mid X_{t_{k-1}}, V_{t_{k-1}}\right] e^{(1-\phi) X_{t_{k-1}}}\right]\right|_{\phi=1} \\
& =\left.e^{-X_{0}} \frac{\partial^{2}}{\partial \phi^{2}} E\left[e^{X_{t_{k-1}}+B\left(\Theta ; \Delta t_{k}, \mathbf{q}_{1}\right) V_{t_{k-1}}+\Gamma\left(\Theta ; \Delta t_{k}, \mathbf{q}_{1}\right)+\Lambda\left(\Theta ; \Delta t_{k}, \mathbf{q}_{1}\right)}\right]\right|_{\phi=1} \\
& =\left.\frac{\partial^{2}}{\partial \phi^{2}} e^{B\left(\Theta ; t_{k-1}, \mathbf{q}_{2}\right) V_{0}+\Gamma\left(\Theta ; t_{k-1}, \mathbf{q}_{2}\right)+\Lambda\left(\Theta ; t_{k-1}, \mathbf{q}_{2}\right)}\right|_{\phi=1},
\end{aligned}
$$


where $\mathbf{q}_{1}=\left(\begin{array}{lll}\phi & 0 & 0\end{array}\right)^{T}$, and

$$
\mathbf{q}_{2}=\left(\begin{array}{c}
1 \\
B\left(\Theta ; \Delta t_{k}, \mathbf{q}_{1}\right) \\
\Gamma\left(\Theta ; \Delta t_{k}, \mathbf{q}_{1}\right)+\Lambda\left(\Theta ; \Delta t_{k}, \mathbf{q}_{1}\right)
\end{array}\right)
$$

In the above derivation procedure, we have made use of the analytic form of the joint moment generating function of $X_{t}$ and $V_{t}$. The fair strike price of the gamma swap is then given by

$$
K_{\Gamma}(T, N)=\left.\frac{A}{N} \sum_{k=1}^{N} \frac{\partial^{2}}{\partial \phi^{2}} e^{B\left(\Theta ; t_{k-1}, \mathbf{q}_{2}\right) V_{0}+\Gamma\left(\Theta ; t_{k-1}, \mathbf{q}_{2}\right)+\Lambda\left(\Theta ; t_{k-1}, \mathbf{q}_{2}\right)}\right|_{\phi=1}
$$

which is seen to have no dependence on the initial price level $S_{t_{0}}$.

It is well known that the gamma exposure of vanilla variance swaps is insensitive to the underlying level of share price (Demeterifi et al., 1999), a property known as constant cash gamma exposure. Suppose an investor is interested in the gamma exposure in the number of portfolio units rather than the initial cash value of the portfolio, the gamma swaps provide constant share gamma exposure by choosing weights that are set equal to the underlying level at the sampling dates. The proof of this property of constant (almost constant) share gamma exposure for the continuously (discretely) sampled gamma swaps is shown below.

\section{Constant share gamma exposure}

We would like to compute the share gamma exposure of an in-progress gamma swap at time $t$, where $t_{i-1}<t \leq t_{i}, i \geq 1$. The share gamma is defined to be

$$
\Gamma_{S}=\Gamma S_{t}:=\frac{\partial^{2} V_{t}}{\partial S_{t}^{2}} S_{t}
$$

where $\Gamma$ is the usual greek gamma of a derivative product with value function $V_{t}$. Consider a hedging portfolio $\Pi$ whose differential change of value is given by

$$
\mathrm{d} \Pi=\Delta \mathrm{d} S_{t}+\frac{1}{2} \Gamma\left(\mathrm{d} S_{t}\right)^{2}
$$

Since $\mathrm{d} S_{t}$ is in proportion to the share price $S_{t}$, the "cash gamma" of the derivative $\Gamma S_{t}^{2}$ is in units of dollars. After normalizing the cash gamma by the share price, the resulting quantity $\Gamma S_{t}$ is in units of shares and hence it is called the "share gamma". To compute the share gamma of a discretely sampled gamma swap, it is necessary to find the time- $t$ value of the 
gamma swap as follows:

$$
\begin{aligned}
V_{t}= & e^{-r\left(t_{N}-t\right)}\left\{\frac{A}{N} \sum_{k=1}^{i-1} \frac{S_{t_{k}}}{S_{t_{0}}}\left(\ln \frac{S_{t_{k}}}{S_{t_{k-1}}}\right)^{2}+\frac{A}{N} E_{t}\left[\frac{S_{t_{i}}}{S_{t_{0}}}\left(\ln \frac{S_{t_{i}}}{S_{t_{i-1}}}\right)^{2}\right]\right. \\
& \left.+\frac{A}{N} E_{t}\left[\sum_{k=i+1}^{N} \frac{S_{t_{k}}}{S_{t_{0}}}\left(\ln \frac{S_{t_{k}}}{S_{t_{k-1}}}\right)^{2}\right]-K_{\Gamma}(T, N)\right\} \\
= & e^{-r\left(t_{N}-t\right)}\left\{\frac{A}{N} \sum_{k=1}^{i-1} \frac{S_{t_{k}}}{S_{t_{0}}}\left(\ln \frac{S_{t_{k}}}{S_{t_{k-1}}}\right)^{2}\right. \\
& +\left.\frac{A}{N} \frac{S_{t_{i-1}}}{S_{t_{0}}} \frac{\partial^{2}}{\partial \phi^{2}}\left[\left(\frac{S_{t}}{S_{t_{i-1}}}\right)^{\phi} e^{B\left(\Theta ; t_{i}-t, \mathbf{q}_{1}\right) V_{t}+\Gamma\left(\Theta ; t_{i}-t, \mathbf{q}_{1}\right)+\Lambda\left(\Theta ; t_{i}-t, \mathbf{q}_{1}\right)}\right]\right|_{\phi=1} \\
& \left.+\left.\frac{A}{N} \frac{S_{t}}{S_{t_{0}}} \sum_{k=i+1}^{N} \frac{\partial^{2}}{\partial \phi^{2}} e^{B\left(\Theta ; t_{k-1}-t, \mathbf{q}_{2}\right) V_{t}+\Gamma\left(\Theta ; t_{k-1}-t, \mathbf{q}_{2}\right)+\Lambda\left(\Theta ; t_{k-1}-t, \mathbf{q}_{2}\right)}\right|_{\phi=1}-K_{\Gamma}(T, N)\right\}
\end{aligned}
$$

where $\mathbf{q}_{1}=\left(\begin{array}{lll}\phi & 0 & 0\end{array}\right)^{T}$, and

$$
\mathbf{q}_{2}=\left(\begin{array}{c}
1 \\
B\left(\Theta ; \Delta t_{k}, \mathbf{q}_{1}\right) \\
\Gamma\left(\Theta ; \Delta t_{k}, \mathbf{q}_{1}\right)+\Lambda\left(\Theta ; \Delta t_{k}, \mathbf{q}_{1}\right)
\end{array}\right) .
$$

By differentiating eq. (3.3) with respect to $S_{t}$ twice and multiplying by $S_{t}$, we obtain the share gamma as follows:

$$
\Gamma_{S}=e^{-r\left(t_{N}-t\right)} \frac{A}{N} \frac{2}{S_{t_{0}}}\left[\left(\ln \frac{S_{t}}{S_{t_{i-1}}}+1\right) F(1)+F^{\prime}(1)\right]
$$

where

$$
F(\phi)=e^{B\left(\Theta ; t_{i}-t, \mathbf{q}_{1}\right) V_{t}+\Gamma\left(\Theta ; t_{i}-t, \mathbf{q}_{1}\right)+\Lambda\left(\Theta ; t_{i}-t, \mathbf{q}_{1}\right)} .
$$

The dependence of $\Gamma_{S}$ on $S_{t}$ appears in the term $\ln \frac{S_{t}}{S_{t_{i-1}}}$. Suppose we take the limit of continuous sampling, $S_{t} \rightarrow S_{t_{i-1}}$, we then obtain

$$
\Gamma_{S} \rightarrow e^{-r\left(t_{N}-t\right)} \frac{A}{N} \frac{2}{S_{t_{0}}}
$$

The above limit has no dependence on $S_{t}$, so it verifies the property of constant gamma exposure under continuous sampling. A similar result has been obtained by Jacquier and Slaoui (2010) using a replication argument.

\section{Fair strike prices of continuously monitored gamma swaps}

By following a similar procedure of taking the limit of vanishing sampling time interval (see Appendix B), we can obtain the following closed form formula for the fair strike price of a continuously monitored gamma swap

$$
K_{\Gamma}(T, \infty)=\frac{1}{T}\left[\left(V_{0}-\frac{\kappa \theta}{\kappa-\rho \varepsilon}-C_{2}\right) \frac{e^{(r-d-\kappa+\rho \varepsilon) T}-1}{r-d-\kappa+\rho \varepsilon}+\left(\frac{\kappa \theta}{\kappa-\rho \varepsilon}+C_{1}+C_{2}\right) \frac{e^{(r-d) T}-1}{r-d}\right]
$$


where

$$
\begin{aligned}
C_{1} & =\frac{\lambda e^{\nu+\delta^{2} / 2}}{1-\rho_{J} \eta}\left[\left(\nu+\delta^{2}+\frac{\rho_{J} \eta}{1-\rho_{J} \eta}\right)^{2}+\delta^{2}+\left(\frac{\rho_{J} \eta}{1-\rho_{J} \eta}\right)^{2}\right] \\
C_{2} & =\frac{\lambda \eta e^{\nu+\delta^{2} / 2}}{\left(1-\rho_{J} \eta\right)^{2}(\kappa-\rho \varepsilon)} .
\end{aligned}
$$

\subsection{Corridor variance swaps}

A corridor variance swap differs from the vanilla variance swap in that the underlying price must fall inside a specified corridor $(L, U](L \geq 0, U<\infty)$, in order for its squared return to be included in the floating leg of the corridor variance swap. For a discretely sampled corridor variance swap with the tenor $0=t_{0}<t_{1}<t_{2}<\cdots<t_{N}=T$, suppose the corridor is

monitored on the underlying price at the old time level $t_{k-1}$ for the $k^{\text {th }}$ squared log return, the floating leg with the corridor $(L, U]$ is given by

$$
\frac{A}{N} \sum_{k=1}^{N}\left(\ln \frac{S_{t_{k}}}{S_{t_{k-1}}}\right)^{2} \mathbf{1}_{\left\{L<S_{t_{k-1}} \leq U\right\}} .
$$

Here, $\mathbf{1}_{\{\cdot\}}$ denotes the indicator function. Corridor variance swaps with a one-sided barrier are also widely traded in the financial markets, where the downside-variance swap and upside-variance swap can be obtained by taking $L=0$ and $U \rightarrow \infty$, respectively. As further generalizations, one can choose to have the corridor monitored on the underlying price at the new time level $t_{k}$ (Sepp, 2007) or even at both time levels (Carr and Lewis, 2004).

Corridor variance swaps allow the investors to take their views on the implied volatility skew. Suppose the implied volatility skew is expected to steepen, the investor may benefit from buying a downside-variance swap and selling an upside-variance swap if this view is realized. Also, investors seeking crash protection may buy the downside-variance swap since it can provide almost the same level of crash protection as the vanilla variance swap but at a lower premium.

It suffices to consider pricing downside-variance swaps alone since the payoffs of downsidevariance swaps of varying values of the upper barrier are sufficient to span all different payoffs of various corridor variance swaps. We would like to find the fair strike price of a downsidevariance swap with an upper barrier $U$ whose payoff at maturity $T$ is given by

$$
\frac{A}{N} \sum_{k=1}^{N}\left(\ln \frac{S_{t_{k}}}{S_{t_{k-1}}}\right)^{2} \mathbf{1}_{\left\{S_{t_{k-1}} \leq U\right\}}-K
$$


Let us consider the expectation calculation of a typical term:

$$
\begin{aligned}
& E\left[\left(\ln \frac{S_{t_{k}}}{S_{t_{k-1}}}\right)^{2} \mathbf{1}_{\left\{S_{t_{k-1}} \leq U\right\}}\right] \\
= & \left.E\left[E\left[\frac{\partial^{2}}{\partial \phi^{2}} e^{\phi\left(X_{t_{k}}-X_{t_{k-1}}\right)} \mid X_{t_{k-1}}, V_{t_{k-1}}\right] \mathbf{1}_{\left\{X_{t_{k-1}} \leq \ln U\right\}}\right]\right|_{\phi=0} \\
= & \left.E\left[\frac{\partial^{2}}{\partial \phi^{2}} e^{B\left(\Theta ; \Delta t_{k}, \mathbf{q}_{1}\right) V_{t_{k-1}}+\Gamma\left(\Theta ; \Delta t_{k}, \mathbf{q}_{1}\right)+\Lambda\left(\Theta ; \Delta t_{k}, \mathbf{q}_{1}\right)} \mathbf{1}_{\left\{X_{t_{k-1}} \leq \ln U\right\}}\right]\right|_{\phi=0} \\
= & \left.\frac{\partial^{2}}{\partial \phi^{2}} E\left[e^{B\left(\Theta ; \Delta t_{k}, \mathbf{q}_{1}\right) V_{t_{k-1}}+\Gamma\left(\Theta ; \Delta t_{k}, \mathbf{q}_{1}\right)+\Lambda\left(\Theta ; \Delta t_{k}, \mathbf{q}_{1}\right)} \mathbf{1}_{\left\{X_{t_{k-1}} \leq \ln U\right\}}\right]\right|_{\phi=0},
\end{aligned}
$$

where $\mathbf{q}_{1}=\left(\begin{array}{lll}\phi & 0 & 0\end{array}\right)^{T}$. For $k=1$, the above expectation is readily seen to be

$$
E\left[\left(\ln \frac{S_{t_{1}}}{S_{t_{0}}}\right)^{2} \mathbf{1}_{\left\{S_{t_{0}} \leq U\right\}}\right]=\left.\frac{\partial^{2}}{\partial \phi^{2}} e^{B\left(\Theta ; \Delta t_{1}, \mathbf{q}_{1}\right) V_{0}+\Gamma\left(\Theta ; \Delta t_{1}, \mathbf{q}_{1}\right)+\Lambda\left(\Theta ; \Delta t_{1}, \mathbf{q}_{1}\right)} \mathbf{1}_{\left\{X_{0} \leq \ln U\right\}}\right|_{\phi=0} .
$$

For $k \geq 2$, the evaluation of expectation in formula (3.6) requires the representation of the indicator function $\mathbf{1}_{\left\{X_{t_{k-1}} \leq \ln U\right\}}$ in terms of an inverse Fourier transform integral. As a result, formula 3.6 can be expressed in terms of a Fourier integral as follows:

$$
\begin{aligned}
& E\left[\left(\ln \frac{S_{t_{k}}}{S_{t_{k-1}}}\right)^{2} \mathbf{1}_{\left\{S_{t_{k-1}} \leq U\right\}}\right] \\
= & \frac{e^{w_{i}\left(X_{0}-u\right)}}{\pi} \int_{0}^{\infty} \operatorname{Re}\left(e^{-\mathrm{i} w_{r}\left(X_{0}-u\right)} \frac{F_{k}\left(w_{r}+\mathrm{i} w_{i}\right)}{\mathrm{i} w_{r}-w_{i}}\right) \mathrm{d} w_{r}, \quad k \geq 2,
\end{aligned}
$$

where $w=w_{r}+\mathrm{i} w_{i}, u=\ln U$, and

$$
F_{k}(w)=\left.\frac{\partial^{2}}{\partial \phi^{2}} e^{B\left(\Theta ; t_{k-1}, \mathbf{q}_{2}\right) V_{0}+\Gamma\left(\Theta ; t_{k-1}, \mathbf{q}_{2}\right)+\Lambda\left(\Theta ; t_{k-1}, \mathbf{q}_{2}\right)}\right|_{\phi=0}, \quad k \geq 2,
$$

with

$$
\mathbf{q}_{2}=\left(\begin{array}{c}
-\mathrm{i} w \\
B\left(\Theta ; \Delta t_{k}, \mathbf{q}_{1}\right) \\
\Gamma\left(\Theta ; \Delta t_{k}, \mathbf{q}_{1}\right)+\Lambda\left(\Theta ; \Delta t_{k}, \mathbf{q}_{1}\right)
\end{array}\right)
$$

The above Fourier integral is regular provided that $w_{i}$ is appropriately chosen to lie within $(-\infty, 0)$. The proof of eq. $3.7 \mathrm{~b}$ ) is presented in Appendix C. The fair strike price of the downside-variance swap is then given by

$$
\begin{aligned}
K_{D}(T, N)= & \frac{A}{N}\left[\left.\frac{\partial^{2}}{\partial \phi^{2}} e^{B\left(\Theta ; \Delta t_{1}, \mathbf{q}_{1}\right) V_{0}+\Gamma\left(\Theta ; \Delta t_{1}, \mathbf{q}_{1}\right)+\Lambda\left(\Theta ; \Delta t_{1}, \mathbf{q}_{1}\right)} \mathbf{1}_{\left\{X_{0} \leq \ln U\right\}}\right|_{\phi=0}\right. \\
& \left.+\frac{e^{w_{i}\left(X_{0}-u\right)}}{\pi} \int_{0}^{\infty} \operatorname{Re}\left(e^{-\mathrm{i} w_{r}\left(X_{0}-u\right)} \frac{\sum_{k=2}^{N} F_{k}\left(w_{r}+\mathrm{i} w_{i}\right)}{\mathrm{i} w_{r}-w_{i}}\right) \mathrm{d} w_{r}\right] .
\end{aligned}
$$


The evaluation of the Fourier integral in eq. 3.8 can be effected by adopting the fast Fourier transform (FFT) algorithm. Actually, by following a similar FFT calculation approach as in Carr and Madan (1999), one can produce the fair strike prices for all downside-variance swaps with varying values of the upper barrier using one single FFT calculation.

\section{An alternative definition of the corridor variance}

We have considered the discretely sampled downside-variance swaps with the breaching of the downside corridor $(0, U]$ being monitored on the stock price at the old time level. However, there is an alternative definition in the literature, where the floating leg payoff is defined to be

$$
\frac{A}{N} \sum_{k=1}^{N}\left(\ln \frac{S_{t_{k}}}{S_{t_{k-1}}}\right)^{2} \mathbf{1}_{\left\{S_{t_{k}} \leq U\right\}}
$$

In this new definition, the breaching of the downside corridor for the $k^{\text {th }}$ squared log return is monitored on the stock price at the new time level. For $k=1,2, \cdots, N$, by following a similar procedure as shown in eq. (3.6), we manage to obtain

$$
E\left[\left(\ln \frac{S_{t_{k}}}{S_{t_{k-1}}}\right)^{2} \mathbf{1}_{\left\{S_{t_{k}} \leq U\right\}}\right]=\frac{e^{w_{i}\left(X_{0}-u\right)}}{\pi} \int_{0}^{\infty} \operatorname{Re}\left(e^{-\mathrm{i} w_{r}\left(X_{0}-u\right)} \frac{F_{k}\left(w_{r}+\mathrm{i} w_{i}\right)}{\mathrm{i} w_{r}-w_{i}}\right) \mathrm{d} w_{r},
$$

where $w=w_{r}+\mathrm{i} w_{i}, w_{i}$ is chosen to lie in $(-\infty, 0)$ as before, $u=\ln U$, and

$$
F_{k}(w)=\left.\frac{\partial^{2}}{\partial \phi^{2}} e^{B\left(\Theta ; t_{k-1}, \mathbf{q}_{2}\right) V_{0}+\Gamma\left(\Theta ; t_{k-1}, \mathbf{q}_{2}\right)+\Lambda\left(\Theta ; t_{k-1}, \mathbf{q}_{2}\right)}\right|_{\phi=0},
$$

with $\mathbf{q}_{1}=\left(\begin{array}{llll}\phi-\mathrm{i} w & 0 & 0\end{array}\right)^{T}$ and

$$
\mathbf{q}_{2}=\left(\begin{array}{c}
-\mathrm{i} w \\
B\left(\Theta ; \Delta t_{k}, \mathbf{q}_{1}\right) \\
\Gamma\left(\Theta ; \Delta t_{k}, \mathbf{q}_{1}\right)+\Lambda\left(\Theta ; \Delta t_{k}, \mathbf{q}_{1}\right)
\end{array}\right)
$$

In Section 4, we will investigate the impact of the alternative definition on the fair strike price of a downside-variance swap.

\section{Fair strike prices for continuously monitored downside-variance swaps}

By taking the asymptotic limit of vanishing sampling time interval, the fair strike price of the continuously sampled downside-variance swaps is given by (see Appendix B)

$$
K_{D}(T, \infty)=\frac{e^{w_{i}\left(X_{0}-u\right)}}{T \pi} \int_{0}^{\infty} \int_{0}^{T} \operatorname{Re}\left(e^{-\mathrm{i} w_{r}\left(X_{0}-u\right)} \frac{F\left(w_{r}+\mathrm{i} w_{i}, t\right)}{\mathrm{i} w_{r}-w_{i}}\right) \mathrm{d} t \mathrm{~d} w_{r},
$$

where

$$
\begin{aligned}
F(w, t)= & e^{B^{0}(-\mathrm{i} w, t) V_{0}+\Gamma^{0}(-\mathrm{i} w, t)+\Lambda^{0}(-\mathrm{i} w, t)}\left\{B^{1}(-\mathrm{i} w, t) V_{0}+\Gamma^{1}(-\mathrm{i} w, t)+\Lambda^{1}(-\mathrm{i} w, t)\right. \\
& \left.+\lambda\left[\left(\nu+\rho_{J} \eta\right)^{2}+\delta^{2}+\rho_{J}^{2} \eta^{2}\right]\right\}
\end{aligned}
$$

and the coefficient functions $B^{0}\left(\phi, t_{k-1}\right), B^{1}\left(\phi, t_{k-1}\right), \Gamma^{0}\left(\phi, t_{k-1}\right), \Gamma^{1}\left(\phi, t_{k-1}\right), \Lambda^{0}\left(\phi, t_{k-1}\right)$ and $\Lambda^{1}\left(\phi, t_{k-1}\right)$ are defined in Appendix B [see eq. (B.3)]. 


\subsection{Conditional variance swaps}

A conditional variance swap is similar to a corridor variance swap, though they differ in the following two aspects:

(i) The accumulated sum of squared returns is divided by the number of observations $D$ that the underlying asset price stays within the corridor instead of the total number of sampling observations $N$;

(ii) The final payoff to the holder is scaled by the ratio $D / N$.

Let $K$ be the strike price of a conditional downside-variance swap and $K^{\prime}$ be the strike price of its corridor variance swap counterpart. The holder's payoff of the conditional downsidevariance swap with corridor's upper barrier $U$ is given by

$$
\begin{aligned}
& \frac{D}{N}\left[\frac{A}{D} \sum_{k=1}^{N}\left(\ln \frac{S_{t_{k}}}{S_{t_{k-1}}}\right)^{2} \mathbf{1}_{\left\{S_{t_{k-1}} \leq U\right\}}-K\right] \\
= & {\left[\frac{A}{N} \sum_{k=1}^{N}\left(\ln \frac{S_{t_{k}}}{S_{t_{k-1}}}\right)^{2} 1_{\left\{S_{t_{k-1}} \leq U\right\}}-K^{\prime}\right]+\left(K^{\prime}-K \frac{D}{N}\right), }
\end{aligned}
$$

where $D=\sum_{k=1}^{N} \mathbf{1}_{\left\{S_{t_{k-1}} \leq U\right\}}$.

The above formula reveals that the conditional variance swap can be decomposed into a corridor variance swap with the same upper barrier plus a range accrual note. Since we have shown how to find the fair strike price of a downside-variance swap, it suffices to compute the expected number of the sampling dates at which the underlying stock price stays below the upper barrier $U$. For a typical term in $E\left[\sum_{k=1}^{N} \mathbf{1}_{\left\{S_{t_{k-1}} \leq U\right\}}\right]$, the expectation calculation involves

$$
E\left[\mathbf{1}_{\left\{S_{k-1} \leq U\right\}}\right]=E\left[\mathbf{1}_{\left\{X_{k-1} \leq u\right\}}\right], \text { where } u=\ln U .
$$

Following similar calculations as in Sec. 3.2, we obtain

$$
E\left[\mathbf{1}_{\left\{X_{k-1} \leq u\right\}}\right]=\frac{e^{w_{i}\left(X_{0}-u\right)}}{\pi} \int_{0}^{\infty} \operatorname{Re}\left(e^{-\mathrm{i} w_{r}\left(X_{0}-u\right)} \frac{G_{k}\left(w_{r}+\mathrm{i} w_{i}\right)}{\mathrm{i} w_{r}-w_{i}}\right) \mathrm{d} w_{r}, k \geq 2,
$$

where $w_{i} \in(-\infty, 0)$ and

$$
G_{k}(w)=e^{B\left(\Theta ; t_{k-1}, \mathbf{q}_{1}\right) V_{0}+\Gamma\left(\Theta ; t_{k-1}, \mathbf{q}_{1}\right)+\Lambda\left(\Theta ; t_{k-1}, \mathbf{q}_{1}\right)},
$$

with $\mathbf{q}_{1}=\left(\begin{array}{lll}-\mathrm{i} w & 0 & 0\end{array}\right)^{T}$. Similarly, the numerical evaluation of the above Fourier integral can be done via the FFT algorithm. Finally, the fair strike price of the conditional downside-variance swap is given by

$$
\begin{aligned}
& K_{C}(T, N) \\
= & K_{D}(T, N)\left[\frac{\mathbf{1}_{\left\{X_{0} \leq u\right\}}}{N}+\frac{e^{w_{i}\left(X_{0}-u\right)}}{\pi N} \int_{0}^{\infty} \operatorname{Re}\left(e^{-\mathrm{i} w_{r}\left(X_{0}-u\right)} \frac{\sum_{k=2}^{N} G_{k}\left(w_{r}+\mathrm{i} w_{i}\right)}{\mathrm{i} w_{r}-w_{i}}\right) \mathrm{d} w_{r}\right]^{-1} .
\end{aligned}
$$


The payoff of a conditional variance swap counts only the sampling dates at which the realized variance does accumulate (conditional on the underlying price lying within the corridor). Compared to the corridor variance swaps, the conditional variance swaps are structured specifically for investors who would like to be exposed only to volatility risk within a prespecified corridor. In a corridor variance swap, the actual amount of the occupation time that the underlying price falls within the corridor over the whole life of the swap has a significant effect on the profit and loss to its holder. However, the conditional variance swap is immunized from this risk since only the realized variance within the corridor matters. Indeed, the decomposition formula (3.11) shows that the holder of a conditional variance swap receives compensation from the range accrual note when the occupation time attains a lower value leading to a lower payoff in the corridor variance swap counterpart.

\section{Fair strike prices for continuously monitored conditional variance swaps}

The fair strike price of the continuously sampled conditional downside-variance swap is related to that of the downside-variance swap presented in the previous section. The analytical representation formula is given by

$$
K_{C}(T, \infty)=K_{D}(T, \infty)\left[\frac{e^{w_{i}\left(X_{0}-u\right)}}{\pi T} \int_{0}^{\infty} \int_{0}^{T} \operatorname{Re}\left(e^{-\mathrm{i} w_{r}\left(X_{0}-u\right)} \frac{G\left(w_{r}+\mathrm{i} w_{i}, t\right)}{\mathrm{i} w_{r}-w_{i}}\right) \mathrm{d} t \mathrm{~d} w_{r}\right]^{-1},
$$

where

$$
G(w, t)=e^{B\left(\Theta ; t, \mathbf{q}_{1}\right) V_{0}+\Gamma\left(\Theta ; t, \mathbf{q}_{1}\right)+\Lambda\left(\Theta ; t, \mathbf{q}_{1}\right)},
$$

with $\mathbf{q}_{1}=\left(\begin{array}{lll}-\mathrm{i} w & 0 & 0\end{array}\right)^{T}$.

\section{$4 \quad$ Numerical tests}

In this section, we report the numerical calculations that were performed for testing accuracy of the analytic pricing formulas obtained for the various types of discretely sampled generalized variance swaps. We examine the impact of the sampling frequency of the realized variance on the fair strike prices of the discretely sampled variance swaps, gamma swaps, corridor variance swaps and conditional variance swaps, and the convergence of the fair strike prices to those of their continuously monitored counterparts. In particular, we used different sets of model parameters in the numerical calculations in order to have a more comprehensive view of the behavior of convergence to the continuous limits. To show the sensitivity of the fair strike prices of these discretely sampled generalized variance swaps to different model parameters, we provide various plots of the fair strike prices against three key model parameters, namely, correlation coefficient, volatility of variance, and jump intensity. Finally, we investigate the impact of the corridor convention (whether breaching of the corridor is monitored on the stock price at the old time level or new time level) on the pricing of the weekly sampled downsidevariance swaps with varying values of the maturity date. In our numerical examples, we 
adopted the set of parameter values shown in Table 1 that are calibrated to S\&P 500 option prices on November 2, 1993 (Duffie et al., 2000) as our basic set of parameter values. In addition, we take $r=3.19 \%, d=0, S_{0}=1$, and assume the number of trading days in one year to be 252. Unless otherwise stated, we consider one-year swap contracts so that $A=N$ $(T=1)$ and take $U=1$ as the upper barrier of the downside corridor in the corridor and conditional variance swaps.

\begin{tabular}{cc|cc}
\hline$\kappa$ & 3.46 & $\nu$ & -0.086 \\
$\theta$ & $(0.0894)^{2}$ & $\eta$ & 0.05 \\
$\varepsilon$ & 0.14 & $\lambda$ & 0.47 \\
$\rho$ & -0.82 & $\rho_{J}$ & -0.38 \\
$\sqrt{V_{0}}$ & 0.087 & $\delta$ & 0.0001 \\
\hline
\end{tabular}

Table 1: The basic set of parameter values of the SVSJ model.

\subsection{Impact of sampling frequency}

First, we present numerical results that explore the convergence behavior of the fair strike prices of different types of discretely monitored generalized variance swaps under varying sampling frequencies. In Table 2, we report the fair strike prices of the vanilla variance swaps, gamma swaps, downside-variance swaps and conditional downside-variance swaps with varying sampling frequencies and different values of the correlation coefficient $\rho$. The choice of $\rho=-1$ infers the scenario where the market is highly leveraged as the asset return has perfect negative correlation with its variance while the less negative correlation coefficient $\rho=-0.3$ represents the market condition with mild leverage effect. The choice of $\rho=-0.82$ is taken from Table 1 (based on calibration from actual data of option prices). The values of the fair strike prices are all presented in variance points, which is the expected realized variance multiplied by $100^{2}$. The fair strike prices for these discretely sampled generalized variance swaps are numerically calculated using the closed form pricing formulas derived in previous sections [see eqs. (2.7), (3.1), (3.8) and (3.13)]. The fair strike prices of the continuously sampled generalized variance swaps, corresponding to $N=\infty$, are computed using the formulas (2.8), (3.5), (3.10) and (3.14) that are deduced by our asymptotic analysis. It is seen from Table 2 that the fair strike prices of all variance swap products (with the exception of the continuously sampled variance swaps) have dependence on $\rho$. The variance swaps and gamma swaps are seen to be less sensitive to $\rho$ for any sampling frequency specification when compared to the downside-variance and conditional downside-variance swaps. As $N \rightarrow \infty$ (vanishing sampling interval), all the fair strike prices of the discretely sampled generalized variance swaps converge to those of their continuously sampled counterparts. The good agreement between these numerical values of 
fair strike prices provide a check for accuracy of all these analytic pricing formulas. As an interesting observation of the pattern of convergence of the fair strike prices with respect to sampling frequency, the convergence can be from the above or below.

\begin{tabular}{llrrrrrr}
\hline \multicolumn{2}{c}{$\begin{array}{c}\text { Sampling } \\
\text { frequency }\end{array}$} & $\begin{array}{r}N=4 \\
\text { quarterly }\end{array}$ & $\begin{array}{r}N=12 \\
\text { monthly }\end{array}$ & $\begin{array}{r}N=26 \\
\text { biweekly }\end{array}$ & $\begin{array}{r}N=52 \\
\text { weekly }\end{array}$ & $\begin{array}{r}N=252 \\
\text { daily }\end{array}$ & $\begin{array}{r}N=\infty \\
\text { continuous }\end{array}$ \\
\hline Variance & $\rho=-1$ & 187.0839 & 183.4365 & 182.2551 & 181.7172 & 181.2759 & 181.1590 \\
swaps & $\rho=-0.82$ & 186.7823 & 183.3154 & 182.1961 & 181.6870 & 181.2695 & 181.1590 \\
& $\rho=-0.3$ & 185.9113 & 182.9654 & 182.0257 & 181.5998 & 181.2512 & 181.1590 \\
\hline Gamma & $\rho=-1$ & 170.1311 & 169.2752 & 169.2176 & 169.2203 & 169.2350 & 169.2407 \\
swaps & $\rho=-0.82$ & 171.0131 & 169.9908 & 169.8749 & 169.8504 & 169.8426 & 169.8423 \\
& $\rho=-0.3$ & 173.6134 & 172.0962 & 171.8081 & 171.7036 & 171.6293 & 171.6113 \\
\hline Downside & $\rho=-1$ & 111.5139 & 102.5147 & 101.3211 & 101.0009 & 100.8345 & 100.8043 \\
variance & $\rho=-0.82$ & 110.5369 & 101.0294 & 99.6504 & 99.2447 & 99.0083 & 98.9599 \\
swaps & $\rho=-0.3$ & 107.8140 & 96.8144 & 94.8855 & 94.2254 & 93.7809 & 93.6779 \\
\hline Conditional & $\rho=-1$ & 216.8810 & 250.5501 & 265.4668 & 272.9108 & 279.2977 & 281.0162 \\
variance & $\rho=-0.82$ & 213.6660 & 244.5615 & 258.3023 & 265.1702 & 271.0668 & 272.6579 \\
swaps & $\rho=-0.3$ & 204.5881 & 227.7824 & 238.2826 & 243.5650 & 248.1260 & 249.3580 \\
\hline
\end{tabular}

Table 2: Comparison of the numerical values of the fair strike prices of variance swaps, gamma swaps, downside-variance swaps and conditional downside-variance swaps with varying sampling frequencies and different values of the correlation coefficient $\rho$. Here, $N$ is the number of return samples within one year.

To present a better visual view on the convergence behavior of the four types of generalized variance swaps, we show various plots of the percentage difference in the fair strike price against varying values of sampling interval $\Delta t$ (in units of year) for different sets of model parameters (see Figure 1). The percentage difference, as a measurement of the discretization effect, is defined to be $100\left(\frac{K(\Delta t)}{K(0)}-1\right)$. Here, $K(\Delta t)$ is the fair strike price with sampling interval $\Delta t$ and $K(0)$ is the corresponding continuous limit of $\Delta t \rightarrow 0^{+}$. Among the four curves of percentage difference in fair strike plotted for each product, the curve labeled "basic" is obtained based on the basic set of model parameters in Table 1. The curve labeled " $\rho=$ -1 " is computed by changing the parameter value of $\rho$ to -1 while keeping other model parameters unchanged, and similar interpretation for the other two curves labeled " $\varepsilon=0.25$ " and " $\lambda=0.6$ ". It is observed that the impact of sampling frequency on the fair strike prices for most generalized variance swaps is small for the chosen range of $\Delta t$ (the lowest sampling frequency being weekly in the plots). In particular, the fair strike prices of the gamma swaps show the least sensitivity to sampling frequency which may be possibly related to the constant 
share gamma property. An exception to insensitivity to sampling frequency is shown by the conditional downside-variance swap. This is not surprising since its fair strike price is equal to that of a downside-variance swap rescaled by $N / E[D]$, where $E[D]$ is the expected number of observations that the asset price falls within the corridor. The sensitivity to sampling frequency is then somehow enlarged by the rescaling factor.

The plots in Figures 2(a) and 2(d) show that the fair strike prices of the vanilla variance swaps and conditional downside-variance swaps exhibit an almost linear rate of convergence. However, careful observation of the plots in Figures 2(b) and 2(c) of the fair strike prices of the gamma swaps and downside-variance swaps reveal some convex curvature in the curves. This convexity behavior is not in good agreement with the results reported in the literature. The numerical tests performed by Broadie and Jain (2008) reveal a linear rate of convergence of vanilla variance swaps under various stochastic volatility models of the asset price process. Under the time-changed Lévy processes, Crosby and Davis (2011) manage to establish mathematically (under certain assumptions) the linear rate of convergence of various generalized variance swaps. However, due to the limitation of their approach, they have neither been able to perform a similar analysis for the generalized variance swaps with corridor restriction on the realized variance, nor make the same conclusion under the general asset price dynamics, say the SVSJ model. Our numerical results provide exceptions that the property of the linear convergence may not be always valid for discretely sampled generalized variance swaps.

\subsection{Sensitivity of fair strike prices to key model parameters}

Next, we examine the sensitivity of the fair strike price of various weekly sampled generalized variance swaps on the following key model parameters: (i) correlation coefficient $\rho$, (ii) volatility of variance $\varepsilon$, (iii) jump intensity $\lambda$. The comparison of the fair strike prices of various generalized variance swaps with varying values of the above model parameters are shown in Figure 2. These plots reveal the different degrees of impact on the fair strike prices of different types of generalized variance swaps with respect to these three model parameters.

Figure 2(a) shows that the fair strike price of the conditional downside-variance swap has the highest sensitivity to $\rho$, followed by that of the downside-variance swap. Moreover, the fair strike prices of the downside-variance swap and conditional downside-variance swap tend to increase as $\rho$ becomes more negative. To explain this phenomenon by an intuitive argument, we observe that when the leverage effect becomes stronger it is more likely that the spike of volatility is accompanied by a plunge in the asset price. The fair strike prices of the variance swap and gamma swap exhibit less sensitivity to $\rho$. For the variance swap, insensitivity to $\rho$ is not surprising since its continuously sampled counterpart has no dependence on $\rho$. In the event of volatility running high, the gamma swap assigns lower weights to the sampled values of 
higher realized variance due to the decline in the underlying asset price in view of the negative correlation. This explains the slight drop in the fair strike price of the gamma swap when $\rho$ becomes more negative.

Figure 2(b) shows that the fair strike price of the conditional downside-variance swap is most sensitive to $\varepsilon$, followed by that of the downside-variance swap. Normally, we expect that a higher $\varepsilon$ would lead to a higher level of accumulation of the realized variance as in the case of the downside-variance swap or conditional downside-variance swap. Again, the vanilla variance swap exhibits insensitivity to $\varepsilon$ as the fair strike price of its continuously sampled counterpart does not depend on $\varepsilon$. The dependence of the fair strike price on $\varepsilon$ for the gamma swap is reversed, which may be attributed to the use of a negative value of correlation in the numerical calculations. Given a negative correlation, it is more likely for the asset price to sink when the variance process is more volatile.

Various earlier papers [e.g. Broadie and Jain (2008)] report the strong dependence of the fair strike prices of variance swaps on jumps. The jumps in the underlying price and variance under the SVSJ model can be characterized by a set of jump parameters, namely, $\lambda, \nu, \eta, \rho_{J}$ and $\delta$. The jump intensity $\lambda$ is considered to be the most crucial parameter. In our calculations, we take $\nu$ and $\rho_{J}$ to be negative. As a result, each jump would most likely lead to a decline in the underlying asset price. Actually, a larger value of $\lambda$ leads to a higher chance of crash in the underlying asset price. Figure 2(c) shows how the fair strike prices of various swap products change with varying values of $\lambda$. All these four types of swap products are seen to show high sensitivity to $\lambda$. The conditional downside-variance swap is most sensitive to $\lambda$, followed by the variance swap, gamma swap and then downside-variance swap. The least sensitivity to $\lambda$ of the downside-variance swap may be attributed to an offsetting effect since a spike of the underlying asset price may result from a potential positive jump.

\subsection{Impact of upper barrier of corridor and breaching convention on downside-variance swaps}

Lastly, we investigate the impact on the fair strike prices of the discretely sampled downsidevariance swaps with varying values of the corridor's upper barrier and different maturities.

In Figures $3(a, b)$, we show the plot of the fair strike price of the weekly sampled downsidevariance swap with varying values of the corridor's upper barrier $U$ and different maturities. The plots shown in Figures $3(a, b)$ reveal the significant impact of the choice of upper barrier $U$ on the fair strike prices of the downside-variance swaps, in particular when $U$ is chosen close to the current stock price $S_{0}$ (inferred from the steep slopes there). The difference in the fair strike prices of the two different types of downside-variance swaps, corresponding to the corridor's upper barrier $U$ being monitored on the stock price at the old time level ("convention 1") or 
new time level ("convention 2"), can be quite substantial when the upper barrier is close to the current stock price $S_{0}$ (set equal to 1). Moreover, the fair strike prices of the swap contracts with the corridor monitored at the new time level are consistently larger than those of the swap contracts having the corridor monitored at the old time level. The difference in the fair strike prices vanishes when $U$ is set extremely low or high. For downside-variance swaps with shorter maturity of half a year, the difference in the fair strike prices based on the two different breaching conventions can be more profound.

\section{Conclusion}

In this paper, we demonstrate an analytic approach of deriving closed form pricing formulas of various types of discretely sampled generalized variance swaps under the dynamics of stochastic volatility with simultaneous jumps in the underlying asset price and its variance. The success of the analytic approach relies on the availability of the analytic expression of the joint moment generating function of the SVSJ model. We manage to derive analytic pricing formulas for the gamma swaps, corridor variance swaps, and conditional variance swaps. The last two types of generalized variance swaps with corridor constraints have their terminal payoffs dependent on the stochastic occupation time during which the underlying asset price lies within a specified corridor. The semi-analytic pricing formulas for the corridor and conditional variance swaps are expressed in terms of Fourier integrals. The numerical evaluation of these Fourier integrals can be performed effectively, thanks to the fast Fourier transform algorithm. We also demonstrate through analytic asymptotic analysis that the pricing formulas of the discretely monitored generalized variance swaps converge to those of their continuously monitored counterparts. Though this paper is focused on pricing discretely monitored generalized variance swaps under the SVSJ framework, the analytic procedure can be applied to any affine model of the underlying asset price and payoff structures of higher moments swaps.

We performed numerical evaluation of these pricing formulas to examine the impact of sampling frequency on the fair strike prices of the gamma swaps, corridor variance swaps and conditional variance swaps. We present various plots that demonstrate the convergence of the fair strike prices of the discretely monitored generalized variance swaps to those of their continuously monitored counterparts, and illustrate the sensitivity of the fair strike price to different choices of model parameter values in the SVSJ model. The fair strike prices of these generalized variance swaps are seen to be more sensitive to the contractual terms in the swap contracts and the choices of model parameter values. For example, there may exist significant difference in the fair strike prices of the downside-variance swaps with respect to whether the stock price of the old time level or new time level is used when the corridor feature is monitored. Our studies show that the impact of sampling frequency on the fair strike price 
can be quite insignificant for some types of generalized variance swaps, like the gamma swaps. Interestingly, the convergence of the fair strike prices with vanishing sampling interval to those of the continuously monitored counterparts can be from the above or below and the linear convergence property may not be always valid for discretely sampled generalized variance swaps. 


\section{References}

Bouzoubaa M., A. Osseiran (2010): Exotic options and hybrids: A guide to structuring, pricing and trading. Wiley, p.250-254.

Broadie, M., A. Jain (2008): The effect of jumps and discrete sampling on volatility and variance swaps. International Journal of Theoretical and Applied Finance, vol. 11(8), p.761797.

Brockhaus, O., D. Long (2000): Volatility swaps made simple. Risk, Jan. issue, p.92-95.

Carr, P., R. Lee (2009): Volatility derivatives. Annual Review of Financial Economics, vol. 1, p.319-339.

Carr, P., K. Lewis (2004): Corridor variance swaps. Risk, Feb. issue, p.67-72.

Carr, P., D. Madan (1998): Towards a theory of volatility trading. In R. Jarrow (ed.), Volatility (London: Risk Books), p.417-427.

Carr, P., D. Madan (1999): Option valuation using the fast Fourier transform. Journal of Computational Finance, vol. 2(4) summer.

Carr. P., L. Wu (2006): A tale of two indices. Journal of Derivatives, vol. 13, p.13-29.

Carr, P., L. Wu (2007): Theory and evidence on dynamic interactions between sovereign credit default swaps and currency options. Journal of Banking and Finance, vol. 31, p.2383-2403.

Chacko, G., S. Das (2002): Pricing interest rate derivatives: a general approach. Review of Financial Studies, vol. 15(1), p.195-241.

Cont, R., T. Kokholm (2008): A consistent pricing model index optons and volatility derivatives. Working paper of the Finance Reseach Group, Aarhus University.

Crosby, J., M.H. Davis (2011): Variance derivatives: pricing and convergence. Working paper of Imperial College, London.

Demeterfi, K., E. Derman, M. Kamal, J. Zou (1999): A guide to volatility and variance swaps. Journal of Derivatives, summer issue, p.9-32.

Drimus, G.G., W. Farkas (2010): Options on discretely sampled variance: discretization effect and greeks. Working paper of University of Copenhagen and University of Zurich.

Duffie D., J. Pan, K. Singleton (2000): Transform analysis and option pricing for affine jumpdiffusions. Econometrica, vol. 68, p.1343-1376. 
Itkin, A., P. Carr (2010): Pricing swaps and options on quadratic variation under stochastic time change models - discrete observations case. Review of Derivatives Research, vol. 13, p.141-176.

Kangro, R., K. Parna, A. Sepp (2004): Pricing European-style options under jump diffusion processes with stochastic volatility: Applications of Fourier transform. Working paper of Tartu University.

Keller-Ressel, M., J. Muhle-Karbe (2010): Asymptotic and exact pricing of options on variance. Working paper of ETH, Zürich.

Jacquier, A., S. Slaoui (2010): Variance dispersion and correlation swaps. Working paper of Imperial College London.

Lee, R. (2010): Gamma swap and corridor variance swap. Encyclopedia of Quantitiative Finance, Wiley.

Lian, G. (2010): Pricing volatility derivatives with stochastic volatility. PhD thesis, University of Wollongong.

Little, T., Pant, V. (2000): A finite-difference method for the valuation of variance swaps. Journal of Computational Finance, vol. 5(1), p.81-101.

Neuberger, A. (1994): The log contract. Journal of Portfolio Management, p.74-80.

Sepp, A. (2007): Variance swaps under no conditions. Risk, Jan. issue, p.82-87.

Sepp, A. (2008): Pricing options on realized variance in the Heston model with jumps in returns and volatility. Journal of Computational Finance, vol. 11(4), p.33-70.

Sepp, A. (2011): Pricing options on realized variance in the Heston model with jumps in returns and volatility II: an approximation of the discrete variance. To appear in Journal of Computational Finance.

Todorov, V., G. Tauchen (2010): Volatility jumps. Working paper of Northwestern University. Windcliff, H., P.A. Forsyth, K.R. Vetzal (2006): Pricing methods and hedging strategies for volatility derivatives. Journal of Banking and Finance, vol. 30, p.409-431.

Zhu, S., G. Lian (2011): A closed-form exact solution for pricing variance swaps with stochastic volatility. Mathematical Finance, vol. 21(2), p.233-256.

\section{Acknowledgement}

This work was supported by the Hong Kong Research Grants Council under Project 642110 of the General Research Funds. 


\section{Appendix A Derivation of eq. (2.6)}

\section{A.1 Solution for $B(\tau):=B(\Theta ; \tau, \mathbf{q})$}

Note that $B(\tau)$ is governed by a non-linear differential equation. To find the solution, we introduce the following transformation, where

$$
B(\tau)=-\frac{2}{\varepsilon^{2}} \frac{E^{\prime}(\tau)}{E(\tau)},
$$

which leads to the following linear differential equation

$$
E^{\prime \prime}(\tau)+(\kappa-\rho \varepsilon \phi) E^{\prime}(\tau)-\frac{1}{4} \varepsilon^{2}\left(\phi-\phi^{2}\right) E(\tau)=0 .
$$

The initial condition $B(0)=b$ gives the derived initial condition for $E(\tau)$, where $E^{\prime}(0)=$ $-\frac{\varepsilon^{2} b}{2} E(0)$. Solving the equation for $E(\tau)$, we obtain

$$
E(\tau)=E(0)\left[\frac{\left(\xi_{+}+\varepsilon^{2} b\right)}{2 \zeta} e^{-\frac{1}{2} \xi_{-} \tau}+\frac{\left(\xi_{-}-\varepsilon^{2} b\right)}{2 \zeta} e^{\frac{1}{2} \xi_{+} \tau}\right],
$$

which then gives

$$
B(\tau)=\frac{b\left(\xi_{-} e^{-\zeta \tau}+\xi_{+}\right)-\left(\phi-\phi^{2}\right)\left(1-e^{-\zeta \tau}\right)}{\left(\xi_{+}+\varepsilon^{2} b\right) e^{-\zeta \tau}+\xi_{-}-\varepsilon^{2} b} .
$$

\section{A.2 Solutions for $\Gamma(\tau)$ and $\Lambda(\tau)$}

Given the solution $B(\tau)$, it is relatively easy to obtain $\Gamma(\tau)$ by direct integration as shown below:

$$
\begin{aligned}
\Gamma(\tau) & =\gamma+(r-d) \phi \tau+\kappa \theta \int_{0}^{\tau} B(u) \mathrm{d} u \\
& =\gamma+(r-d) \phi \tau-\frac{2 \kappa \theta}{\varepsilon^{2}} \ln \frac{E(\tau)}{E(0)} \\
& =(r-d) \phi \tau+\gamma-\frac{\kappa \theta}{\varepsilon^{2}}\left[\xi_{+} \tau+2 \ln \frac{\left(\xi_{+}+\varepsilon^{2} b\right) e^{-\zeta \tau}+\xi_{-}-\varepsilon^{2} b}{2 \zeta}\right] .
\end{aligned}
$$

The evaluation of $\Lambda(\tau)$ requires an expectation calculation followed by integration, where

$$
\Lambda(\tau)=-\lambda(m \phi+1) \tau+\lambda \int_{0}^{\tau} E\left[\exp \left(\phi J^{S}+B(u) J^{V}\right)\right] \mathrm{d} u
$$


We employ the iterated expectation as follows:

$$
\begin{aligned}
E\left[\exp \left(\phi J^{S}+B(u) J^{V}\right)\right] & =E\left[E\left[\exp \left(\phi J^{S}+B(u) J^{V}\right)\right] \mid J^{V}\right] \\
& =E\left[\frac{e^{B(u) J^{V}}}{\sqrt{2 \pi} \delta} \int_{-\infty}^{\infty} \exp \left(\phi x-\frac{\left(x-\nu-\rho_{J} J^{V}\right)^{2}}{2 \delta^{2}}\right) \mathrm{d} x\right] \\
& =\exp \left(\phi \nu+\frac{\delta^{2} \phi^{2}}{2}\right) E\left[\exp \left(\left[\rho_{J} \phi+B(u)\right] J^{V}\right)\right] \\
& =\exp \left(\phi \nu+\frac{\delta^{2} \phi^{2}}{2}\right) \int_{0}^{\infty} \frac{1}{\eta} \exp \left(\left[\rho_{J} \phi+B(u)\right] y-\frac{y}{\eta}\right) \mathrm{d} y \\
& =\exp \left(\phi \nu+\frac{\delta^{2} \phi^{2}}{2}\right) \frac{1}{1-\left[\rho_{J} \phi+B(u)\right] \eta},
\end{aligned}
$$

where the final integration step requires the technical condition: $\operatorname{Re}\left(\rho_{J} \phi+B(u)\right) \eta<1$. Since $\eta$ is generally small, this requirement is usually fulfilled. Lastly, we perform the integration as follows:

$$
\int_{0}^{\tau} \frac{1}{1-\left[\rho_{J} \phi+B(u)\right] \eta} \mathrm{d} u=\int_{0}^{\tau} \frac{k_{1} e^{-\zeta u}+k_{2}}{k_{3} e^{-\zeta u}+k_{4}} \mathrm{~d} u=\frac{k_{2}}{k_{4}} \tau-\frac{1}{\zeta}\left(\frac{k_{1}}{k_{3}}-\frac{k_{2}}{k_{4}}\right) \ln \frac{k_{3} e^{-\zeta \tau}+k_{4}}{k_{3}+k_{4}},
$$

where

$$
\begin{aligned}
& k_{1}=\xi_{+}+\varepsilon^{2} b, \quad k_{2}=\xi_{-}-\varepsilon^{2} b, \\
& k_{3}=\left(1-\phi \rho_{J} \eta\right) k_{1}-\eta\left(\phi-\phi^{2}+\xi_{-} b\right), \\
& k_{4}=\left(1-\phi \rho_{J} \eta\right) k_{2}+\eta\left(\phi-\phi^{2}-\xi_{+} b\right) .
\end{aligned}
$$

In actual implementation, it may be necessary to consider the following two degenerate cases. Suppose $k_{3}=0$, then the integral becomes

$$
\int_{0}^{\tau} \frac{1}{1-\left[\rho_{J} \phi+B(u)\right] \eta} \mathrm{d} u=\frac{k_{2}}{k_{4}} \tau-\frac{k_{1}}{k_{4}} \frac{e^{-\zeta \tau}-1}{\zeta}
$$

and when $k_{4}=0$, the integral takes the form

$$
\int_{0}^{\tau} \frac{1}{1-\left[\rho_{J} \phi+B(u)\right] \eta} \mathrm{d} u=\frac{k_{1}}{k_{3}} \tau+\frac{k_{2}}{k_{3}} \frac{e^{\zeta \tau}-1}{\zeta} .
$$

\section{Appendix B Proof of formulas (2.8), (3.5) and (3.10)}

For notational convenience, we write $B_{\Delta t_{k}}$ as $B\left(\Theta ; \Delta t_{k}, \mathbf{q}_{1}\right)$, and similar interpretation for other parameter functions $\Gamma\left(\Theta ; \Delta t_{k}, \mathbf{q}_{1}\right)$ and $\Lambda\left(\Theta ; \Delta t_{k}, \mathbf{q}_{1}\right)$. When $\mathbf{q}_{1}=\left(\begin{array}{lll}\alpha & 0 & 0\end{array}\right)^{T}$, we expand $B_{\Delta t_{k}}$, $\Gamma_{\Delta t_{k}}$ and $\Lambda_{\Delta t_{k}}$ in powers of $\Delta t_{k}$, where

$$
\begin{aligned}
B_{\Delta t_{k}} & =\frac{1}{2}\left(\alpha^{2}-\alpha\right) \Delta t_{k}+O\left(\Delta t_{k}^{2}\right) \\
\Gamma_{\Delta t_{k}} & =(r-d) \alpha \Delta t_{k}+O\left(\Delta t_{k}^{2}\right) \\
\Lambda_{\Delta t_{k}} & =-\lambda(m \alpha+1) \Delta t_{k}+\frac{\lambda e^{\alpha \nu+\delta^{2} \alpha^{2} / 2}}{1-\alpha \rho_{J} \eta} \Delta t_{k}+O\left(\Delta t_{k}^{2}\right) .
\end{aligned}
$$


Also, we write $B_{t_{k-1}}$ as $B\left(\Theta ; t_{k-1}, \mathbf{q}_{2}\right)$, and similar notational interpretation for $\Gamma_{t_{k-1}}$ and $\Lambda_{t_{k-1}}$. Now, we expand $B_{t_{k-1}}, \Gamma_{t_{k-1}}$ and $\Lambda_{t_{k-1}}$ in powers of $B_{\Delta t_{k}}, \Gamma_{\Delta t_{k}}$ and $\Lambda_{\Delta t_{k}}$, and keep only the linear terms. This gives

$$
\left\{\begin{aligned}
B_{t_{k-1}}= & \frac{\left(\phi^{2}-\phi\right)\left(1-e^{-\zeta t_{k-1}}\right)}{\xi_{+} e^{-\zeta t_{k-1}}+\xi_{-}} \\
& +\left\{\frac{\xi_{-} e^{-\zeta t_{k-1}}+\xi_{+}}{\xi_{+} e^{-\zeta t_{k-1}}+\xi_{-}}+\left(\phi^{2}-\phi\right)\left[\frac{\left(1-e^{-\zeta t_{k-1}}\right) \varepsilon}{\xi_{+} e^{-\zeta t_{k-1}}+\xi_{-}}\right]^{2}\right\} B_{\Delta t_{k}} \\
\Gamma_{t_{k-1}}= & (r-d) \phi t_{k-1}-\frac{\kappa \theta}{\varepsilon^{2}}\left(\xi_{+} t_{k-1}+2 \ln \frac{\xi_{+} e^{-\zeta t_{k-1}}+\xi_{-}}{2 \zeta}\right) \\
& +\frac{2 \kappa \theta\left(1-e^{-\zeta t_{k-1}}\right)}{\xi_{+} e^{-\zeta t_{k-1}}+\xi_{-}} B_{\Delta t_{k}}+\Gamma_{\Delta t_{k}}+\Lambda_{\Delta t_{k}} \\
\Lambda_{t_{k-1}}= & \lambda \varepsilon^{2} \exp \left(\phi \nu+\frac{\delta^{2} \phi^{2}}{2}\right)\left[\frac{t_{k-1}}{J_{2}}-\frac{2 \eta}{J_{1} J_{2}} \ln \frac{\xi_{+} J_{1} e^{-\zeta t_{k-1}}+\xi_{-} J_{2}}{2 \varepsilon^{2} \zeta\left(1-\phi \rho_{J} \eta\right)}\right] \\
& \left.-\lambda(m \phi+1) t_{k-1}\right] \\
& -\frac{2 \eta \lambda \varepsilon^{2}}{J_{1} J_{2}} \exp \left(\phi \nu+\frac{\delta^{2} \phi^{2}}{2}\right)\left[\frac{\varepsilon^{2}\left(J_{1} e^{-\zeta t_{k-1}}-J_{2}\right)}{\xi_{+} J_{1} e^{-\zeta t_{k-1}}+\xi_{-} J_{2}}+\frac{\eta}{1-\phi \rho_{J} \eta}\right] B_{\Delta t_{k}},
\end{aligned}\right.
$$

where $\zeta, \xi_{+}$and $\xi_{-}$are defined in eq. 2.6 and

$$
J_{1}=\left(1-\phi \rho_{J} \eta\right) \varepsilon^{2}-\eta \xi_{-}, J_{2}=\left(1-\phi \rho_{J} \eta\right) \varepsilon^{2}+\eta \xi_{+} .
$$

In the above expansion procedure, we manage to maintain first order accuracy with respect to $\Delta t_{k}$. The second order derivative of $\exp \left(B_{t_{k-1}} V_{0}+\Gamma_{t_{k-1}}+\Lambda_{t_{k-1}}\right)$ [see eq. 2.7]] with respect to $\alpha$ can be expressed as

$$
\begin{aligned}
& \frac{\partial^{2}}{\partial \alpha^{2}} e^{B_{t_{k-1}} V_{0}+\Gamma_{t_{k-1}}+\Lambda_{t_{k-1}}} \\
= & e^{B_{t_{k-1}} V_{0}+\Gamma_{t_{k-1}}+\Lambda_{t_{k-1}}}\left(V_{0} \frac{\partial^{2} B_{t_{k-1}}}{\partial \alpha^{2}}+\frac{\partial^{2} \Gamma_{t_{k-1}}}{\partial \alpha^{2}}+\frac{\partial^{2} \Lambda_{t_{k-1}}}{\partial \alpha^{2}}\right)+O\left(\Delta t_{k}^{2}\right) .
\end{aligned}
$$

For the variance swap, we set $\phi=0$ in $B_{t_{k-1}}, \Gamma_{t_{k-1}}$ and $\Lambda_{t_{k-1}}$ in eq. (B.2). By substituting all the relations between $B_{\Delta t_{k}}, \Gamma_{\Delta t_{k}}, \Lambda_{\Delta t_{k}}, B_{t_{k-1}}, \Gamma_{t_{k-1}}$ and $\Lambda_{t_{k-1}}$, and setting $\alpha=0$, we obtain

$$
\begin{aligned}
& \left.\frac{\partial^{2}}{\partial \alpha^{2}} e^{B_{t_{k-1}} V_{0}+\Gamma_{t_{k-1}}+\Lambda_{t_{k-1}}}\right|_{\alpha=0} \\
= & \left\{e^{-\kappa t_{k-1}} V_{0}+\frac{\lambda \eta}{\kappa}\left(1-e^{-\kappa t_{k-1}}\right)+\lambda\left[\delta^{2}+\rho_{J}^{2} \eta^{2}+\left(\nu+\rho_{J} \eta\right)^{2}\right]\right. \\
& \left.+\theta\left(1-e^{-\kappa t_{k-1}}\right)\right\} \Delta t_{k}+O\left(\Delta t_{k}^{2}\right) .
\end{aligned}
$$

The fair strike price of the variance swap with $N$ sampling dates is then given by

$$
\begin{aligned}
K_{V}(T, N)= & \frac{1}{T} \sum_{k=1}^{N}\left\{e^{-\kappa t_{k-1}} V_{0}+\frac{\lambda \eta}{\kappa}\left(1-e^{-\kappa t_{k-1}}\right)+\lambda\left[\delta^{2}+\rho_{J}^{2} \eta^{2}+\left(\nu+\rho_{J} \eta\right)^{2}\right]\right. \\
& \left.+\theta\left(1-e^{-\kappa t_{k-1}}\right)\right\} \Delta t_{k}+O\left(\Delta t^{2}\right),
\end{aligned}
$$


where $\Delta t=\max _{1 \leq k \leq N} \Delta t_{k}$. The principal term in the above expression is a Riemann left sum which converges to formula (2.8) by taking the limit $\Delta t \rightarrow 0$.

For the gamma swap, we set $\phi=1$ in eq. (B.2). By repeating similar calculations as above, we can obtain the pricing formula (3.5).

For notational convenience, we express the relations in eq. (B.1) in terms of the coefficient functions $B^{0}\left(\phi, t_{k-1}\right), B^{1}\left(\phi, t_{k-1}\right), \Gamma^{0}\left(\phi, t_{k-1}\right), \Gamma^{1}\left(\phi, t_{k-1}\right), \Lambda^{0}\left(\phi, t_{k-1}\right)$ and $\Lambda^{1}\left(\phi, t_{k-1}\right)$ as follows:

$$
\left\{\begin{array}{l}
B_{t_{k-1}}=B^{0}\left(\phi, t_{k-1}\right)+B^{1}\left(\phi, t_{k-1}\right) B_{\Delta t_{k}} \\
\Gamma_{t_{k-1}}=\Gamma^{0}\left(\phi, t_{k-1}\right)+\Gamma^{1}\left(\phi, t_{k-1}\right) B_{\Delta t_{k}}+\Gamma_{\Delta t_{k}}+\Lambda_{\Delta t_{k}} \\
\Lambda_{t_{k-1}}=\Lambda^{0}\left(\phi, t_{k-1}\right)+\Lambda^{1}\left(\phi, t_{k-1}\right) B_{\Delta t_{k}} .
\end{array}\right.
$$

To derive the fair strike price of the continuously sampled downside-variance swap, we set $\phi=$ $-\mathrm{i} w$ in eq. (B.2). Again, by repeating a similar asymptotic analysis as above, we obtain the fair strike price of the continuously sampled conditional downside-variance swap in formula (3.10).

\section{Appendix C Proof of eq. (3.7b)}

We consider the generalized Fourier transform of the indicator function $\mathbf{1}_{\left\{X_{t_{k-1}} \leq u\right\}}$ visualized as a function of $u$, where

$$
\int_{-\infty}^{\infty} \mathbf{1}_{\left\{X_{t_{k-1}} \leq u\right\}} e^{-\mathrm{i} u w} \mathrm{~d} u=\int_{X_{t_{k-1}}}^{\infty} e^{-\mathrm{i} u w} \mathrm{~d} u=\frac{e^{-\mathrm{i} X_{t_{k-1}} w}}{\mathrm{i} w} .
$$

Here, the Fourier transform variable $w$ is taken to be complex and we write $w=w_{r}+\mathrm{i} w_{i}$. Provided that $w_{i}$ is appropriately chosen to lie within $(-\infty, 0)$, the above generalized Fourier transform exists. By taking the corresponding generalized inverse Fourier transform, we obtain

$$
\mathbf{1}_{\left\{X_{t_{k-1}} \leq u\right\}}=\frac{1}{2 \pi} \int_{-\infty}^{\infty} e^{\mathrm{i} u w} \frac{e^{-\mathrm{i} X_{t_{k-1}} w}}{\mathrm{i} w} \mathrm{~d} w_{r} .
$$

This analytic representation of the indicator function expressed in terms of a generalized Fourier integral is then substituted into eq. (3.6). By interchanging the order of performing integration with the two operations of differentiation and expectation, we manage to obtain

$$
\begin{aligned}
& E\left[\left(\ln \frac{S_{t_{k}}}{S_{t_{k-1}}}\right)^{2} \mathbf{1}_{\left\{S_{t_{k-1}} \leq U\right\}}\right] \\
= & \left.\frac{1}{2 \pi} \int_{-\infty}^{\infty} \frac{\partial^{2}}{\partial \phi^{2}} E\left[e^{-\mathrm{i} w X_{t_{k-1}}+B\left(\Theta ; \Delta t_{k}, \mathbf{q}_{1}\right) V_{t_{k-1}}+\Gamma\left(\Theta ; \Delta t_{k}, \mathbf{q}_{1}\right)+\Lambda\left(\Theta ; \Delta t_{k}, \mathbf{q}_{1}\right)}\right]\right|_{\phi=0} \frac{e^{\mathrm{i} u w}}{\mathrm{i} w} \mathrm{~d} w_{r} \\
= & \frac{e^{w_{i}\left(X_{0}-u\right)}}{\pi} \int_{0}^{\infty} \operatorname{Re}\left(e^{-\mathrm{i} w_{r}\left(X_{0}-u\right)} \frac{F_{k}\left(w_{r}+\mathrm{i} w_{i}\right)}{\mathrm{i} w_{r}-w_{i}}\right) \mathrm{d} w_{r}, k \geq 2,
\end{aligned}
$$

as shown in eq. $3.7 \mathrm{~b})$. 
(a) variance swaps

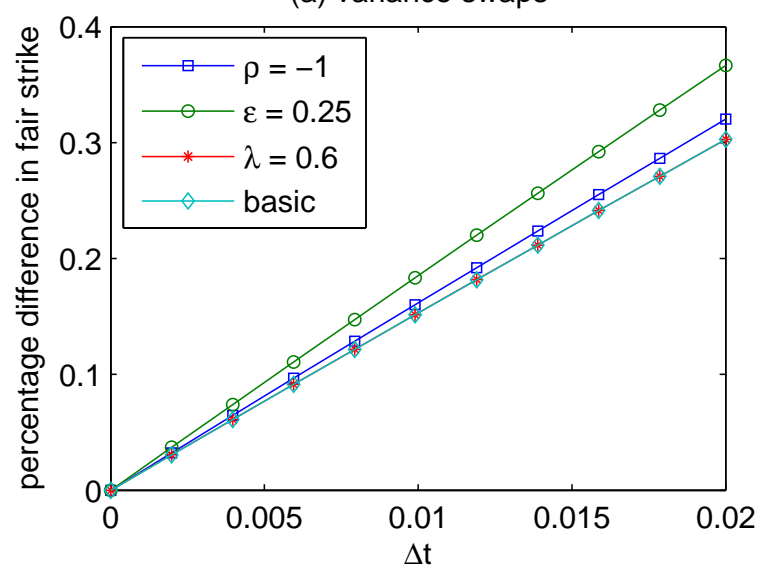

(c) downside-variance swaps

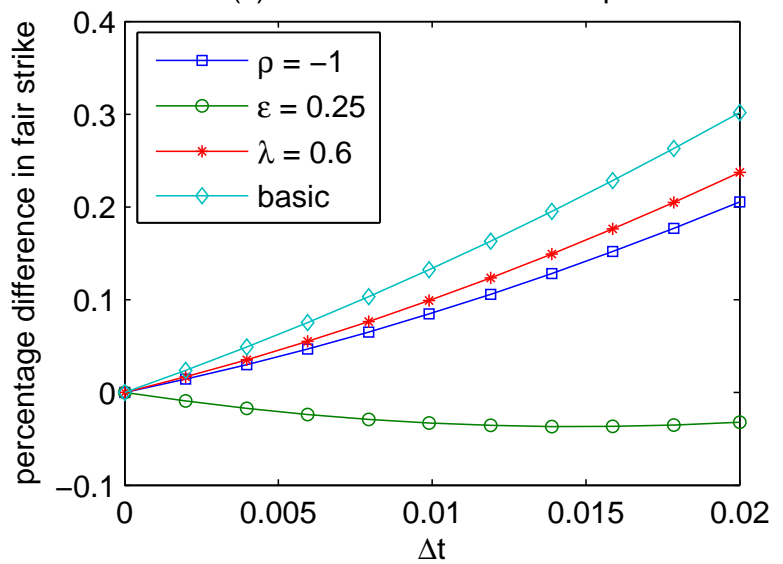

(b) gamma swaps

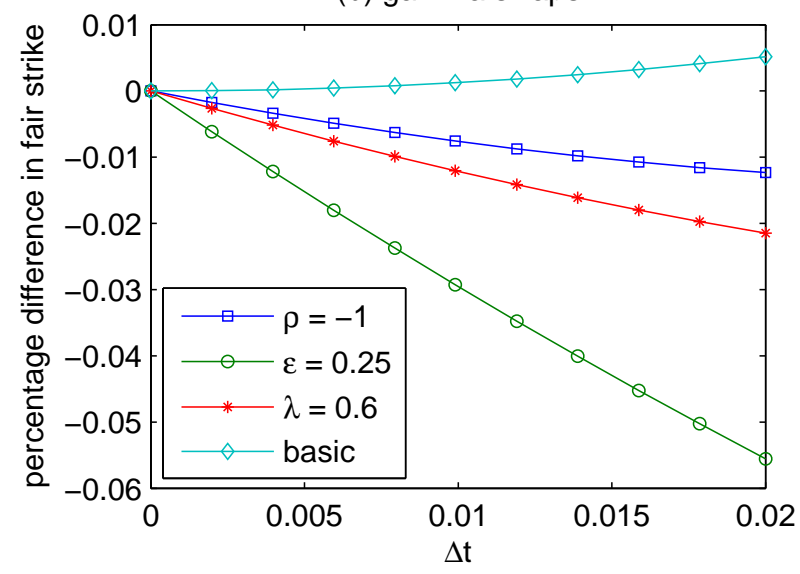

(d) conditional downside-variance swaps

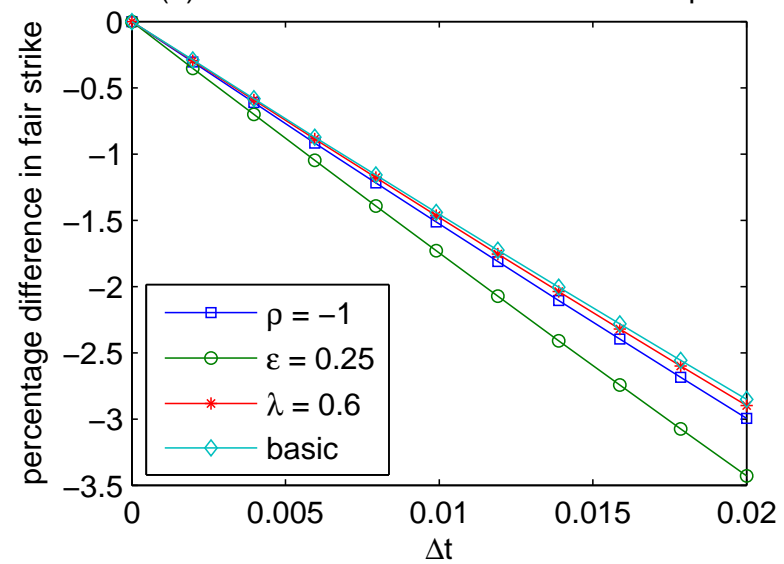

Figure 1: Plot of the percentage difference in the fair strike prices of various discretely sampled generalized variance swaps against sampling time interval $\Delta t$ (in units of year): (a) variance swaps, (b) gamma swaps, (c) downside-variance swaps, (d) conditional downside-variance swaps. The convergence of the fair strike prices to the continuous limit can be from the above or below with vanishing sampling interval. For most cases, the convergence trend is "almost linear" [with a few exceptions, like the curves in Figures 1(b,c)]. 
(a) correlation coefficient, $\rho$

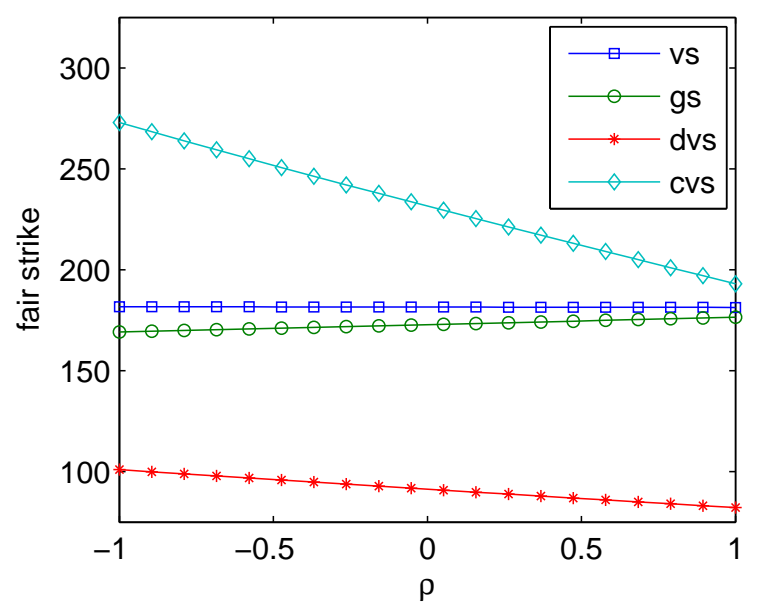

(c) jump intensity, $\lambda$

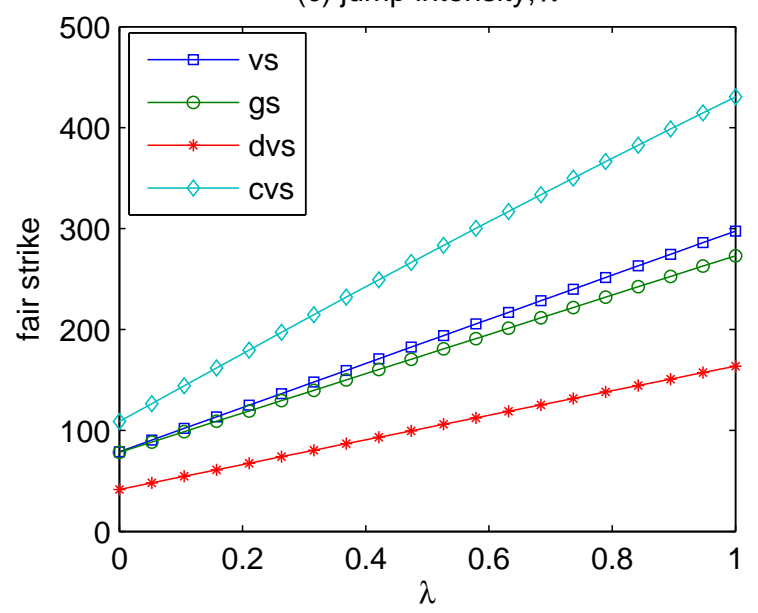

(b) volatility of variance, $\varepsilon$

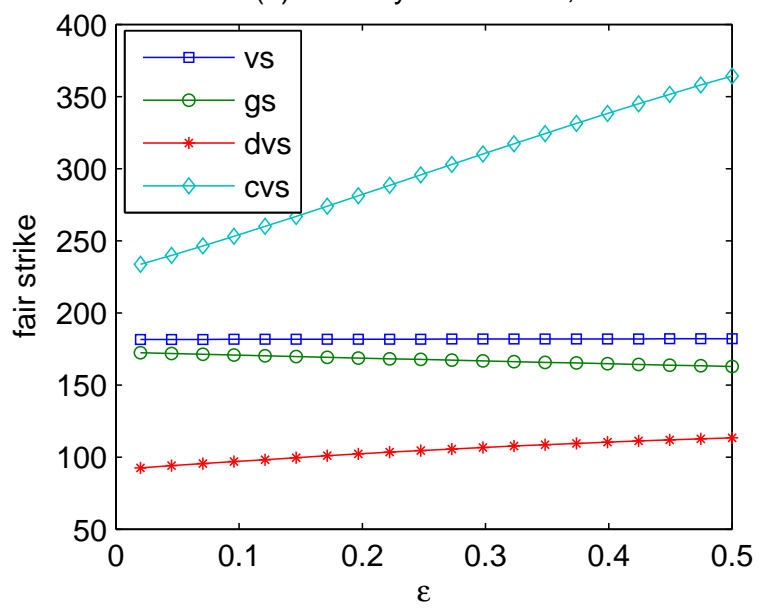

Figure 2: Plot of the fair strike of various discretely sampled generalized variance swaps against (a) correlation coefficient, $\rho$; (b) volatility of variance, $\varepsilon$; (c) jump intensity, $\lambda$. The variance swap products are variance swap (labeled as "vs"), gamma swap (labeled as "gs"), downsidevariance swap (labeled as "dvs") and conditional downside-variance swap (labeled as "cvs"). The fair strike prices of the conditional downside-variance swaps exhibit the highest sensitivity to model parameter values. 
(a) maturity of one year

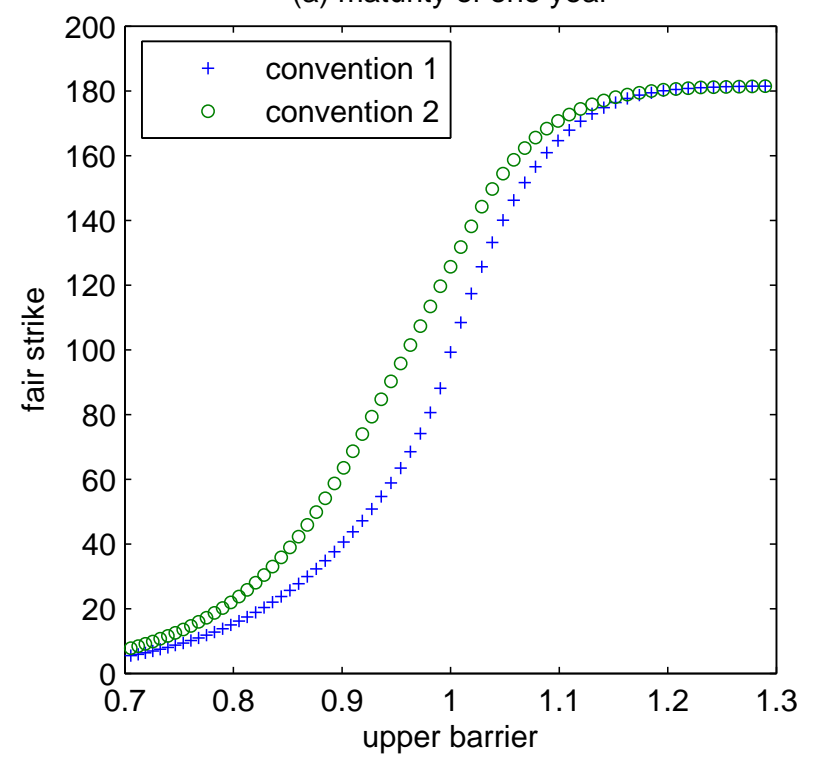

(b) maturity of half a year

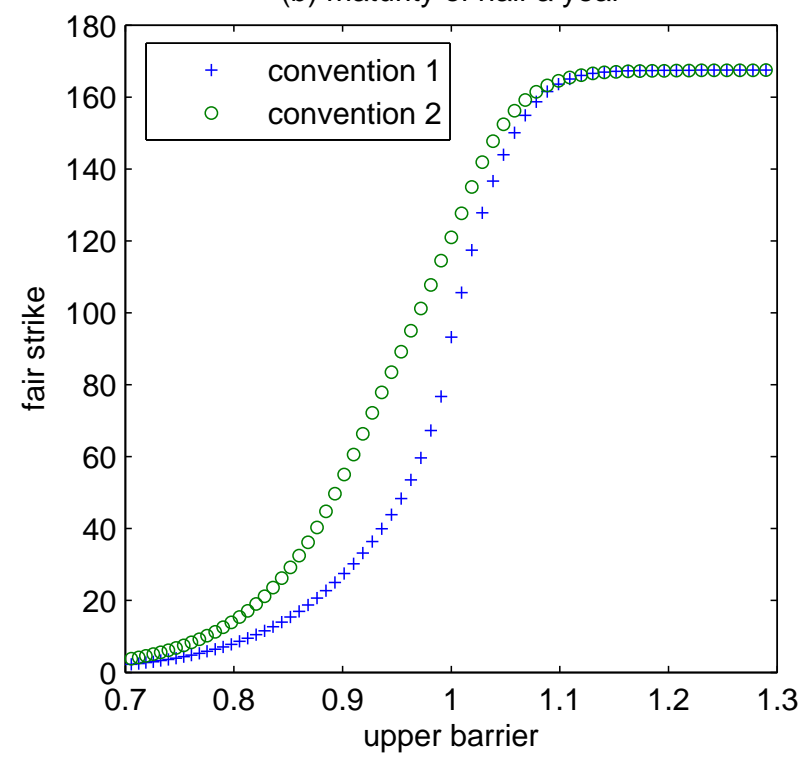

Figure 3: Comparison of the fair strike prices of the weekly sampled downside-variance swaps with varying values of the corridor's upper barrier when breaching of the corridor is monitored on the stock price at the old time level ("convention 1") or new time level ("convention 2"). The difference in the fair strike prices is shown to be more profound for downside-variance swaps with shorter maturities (comparing downside-variance swap contracts with maturities of one year and half a year). 